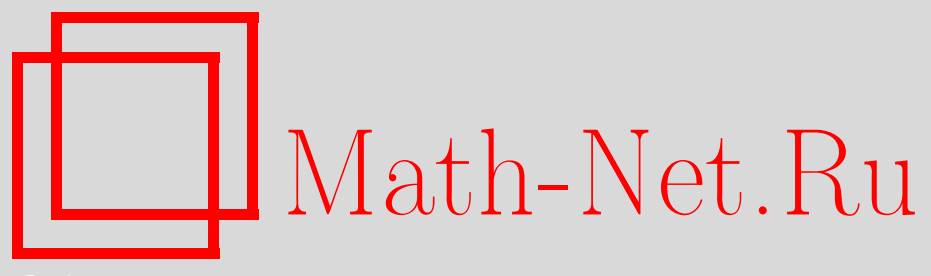

М. Шлихенмайер, О. К. Шейнман, Центральные расширения алгебр операторов Лакса, УМH, 2008, том 63, выпуск 4, 131-172

DOI: https://doi.org/10.4213/rm9221

Использование Общероссийского математического портала Math-Net.Ru подразумевает, что вы прочитали и согласны с пользовательским соглашением http://www . mathnet.ru/rus/agreement

Параметры загрузки:

IP : 107.22 .136 .117

26 апреля 2023 г., 09:08:37

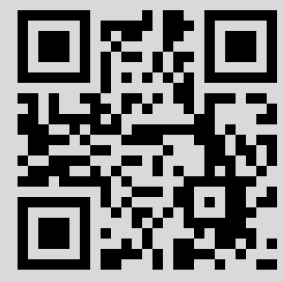




\section{Центральные расширения алгебр операторов Лакса}

\section{М. Шлихенмайер, О.К. Шейнман}

Алгебры операторов Лакса введены И. М. Кричевером и О. К. Шейнманом в развитие теории И. М. Кричевера операторов Лакса на алгебраических кривых. По типу они относятся к почти градуированным алгебрам токов. В настоящей статье дается полная классификация локальных коциклов и почти градуированных центральных расширений алгебр операторов Лакса. Показано, что в случае, когда соответствующая конечномерная алгебра Ли проста, пространство 2-когомологий алгебр операторов Лакса одномерно. При этом важную роль играет действие на алгебре операторов Лакса мероморфных векторных полей, определенное с помощью подходящего ковариантного дифференцирования.

Библиография: 27 названий.

\section{СОДЕРЖАНИЕ}

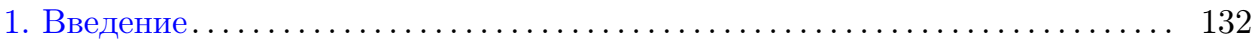

2. Алгебры и структуры на них................................. 137

2.1. Алгебры .................................... 137

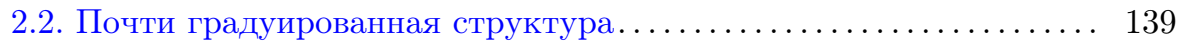

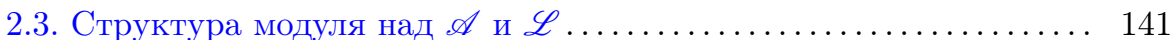

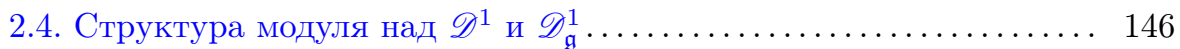

3. Коциклы........................................... 147

3.1. Геометрические коциклы . . . . . . . . . . . . . . . . . . . 147

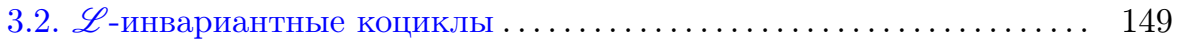

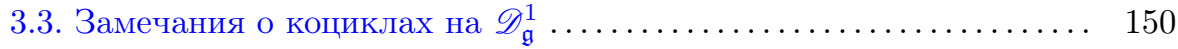

3.4. Локальные коциклы................................ 151

3.5. Основная теорема ................................. 152

4. Единственность $\mathscr{L}$-инвариантных коциклов .................... 154

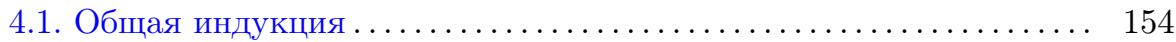

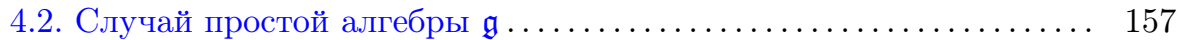

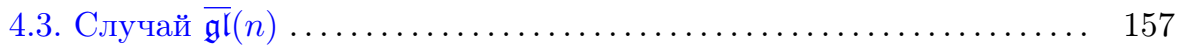

5. Единственность класса когомологий для простого случая ........... 158

Работа выполнена при поддержке гранта R1F10L05 Университета Люксембурга, проекта РФФИ № 08-01-00054-а и программы "Математические методы нелинейной динамики" Президиума РАН.

(C) М. ШлихенмайеР, О. К. ШЕйнман, 2008 


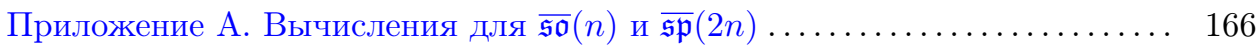

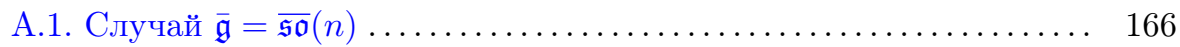

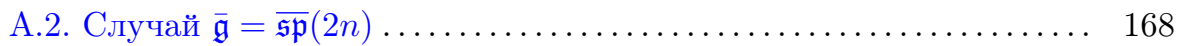

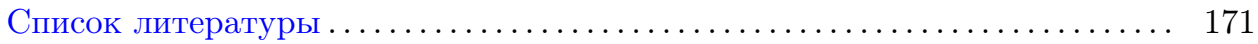

\section{1. Введение}

В настоящей работе мы даем полную классификацию почти градуированных центральных расширений для нового класса одномерных алгебр токов алгебр операторов Лакса. Этому классу принадлежат, в частности, алгебры петель и аффинные алгебры Кричевера-Новикова. Наши результаты дают известную классификацию центральных расширений в этих частных случаях. Мы даем подробный обзор известных к настоящему времени результатов об алгебрах операторов Лакса (за исключением их приложений к интегрируемым системам), обзор результатов и методов в области центральных расширений алгебр токов на одномерных многообразиях, а также краткий обзор некоторых результатов теории интегрируемых систем, к которой рассматриваемый класс алгебр Ли имеет непосредственное отношение.

Алгебры операторов Лакса введены И. М. Кричевером и одним из авторов настоящей работы в [1], где концепция лаксовых операторов на алгебраических кривых, предложенная в [2], обобщена на $\mathfrak{g}$-значные операторы Лакса $(\mathfrak{g}$ - классическая комплексная алгебра Ли). Здесь уместно напомнить, что в [2] известная теория представлений Лакса и нулевой кривизны с рациональным спектральным параметром была обобщена на случай алгебраических кривых $\Sigma$ произвольного рода $g$. Такие представления возникают различными путями в теории интегрируемых систем, например, в [3] введено представление нулевой кривизны со спектральным параметром на эллиптической кривой для уравнения Кричевера-Новикова, а в [2] описан теоретико-полевой аналог системы Калоджеро-Мозера, также на эллиптической кривой. Теория операторов Лакса на римановых поверхностях, предложенная в [2], включает в себя гамильтонову теорию уравнений Лакса и нулевой кривизны, теорию функций Бейкера-Ахиезера и подход к соответствующим алгебро-геометрическим решениям.

Понятие операторов Лакса на алгебраических кривых тесно связано с результатами А.Н. Тюрина о классификации голоморфных расслоений на алгебраических кривых [4]. Оно использует данные Тюрина, моделирующие параметры Тюрина этих расслоений и состоящие из точек $\gamma_{s}(s=1, \ldots, n g)$ и связанных с ними элементов $\alpha_{s} \in \mathbb{C} P^{n-1}$ (где $g$ обозначает род римановой поверхности $\Sigma$, а $n$ - ранг расслоения).

Линейное пространство операторов Лакса, ассоциированное с положительным дивизором $D=\sum_{k} m_{k} P_{k}\left(P_{k} \in \Sigma\right)$, определяется в [2] как пространство мероморфных функций на $\Sigma$, принимающих значения в $(n \times n)$-матрицах и имеющих полюсы кратности не выше $m_{k}$ в точках $P_{k}$ и не более чем простые в точках $\gamma_{s}$. Коэффициенты разложения Лорана такой матричнозначной функции в окрестности точки $\gamma_{s}$ должны удовлетворять определенным линейным ограничениям, зависящим от параметра $\alpha_{s}$ (см. ниже соотношения (2.5)). 
Наблюдение, что лаксовы операторы с полюсами произвольного порядка в точках $P_{k}$ образуют алгебру относительно поточечного умножения, стало отправной точкой работы [1]. В этой работе для $\mathfrak{g}=\mathfrak{s l}(n), \mathfrak{s o}(n), \mathfrak{s p}(2 n)$ над $\mathbb{C}$ были введены g-значные операторы Лакса. Пространство таких операторов образует алгебру Ли по отношению к поточечному коммутированию. Мы обозначаем эту алгебру $\overline{\mathfrak{g}}$. Рассмотрение $\mathfrak{g}$-значных операторов Лакса потребовало определенной модификации вышеупомянутых линейных ограничений. Оказалось даже, что для $\mathfrak{g}=\mathfrak{s p}(2 n)$ порядки полюсов в точках $\gamma_{s}$ следует положить равными 2. Не вызывает сомнения, что путем подходящих модификаций можно построить алгебры операторов Лакса и для других классических алгебр Ли.

С другой стороны, при отсутствии точек $\gamma_{s}$ (что соответствует тривиальному расслоению) мы возвращаемся к известному классу алгебр Кричевера-Новикова (см. обзор в [5], [6]). Если вдобавок род $\Sigma$ равен 0 и носитель $D$ состоит из двух точек, мы получаем (с точностью до изоморфизма) алгебры петель.

Подобно алгебрам Кричевера-Новикова, алгебры операторов Лакса обладают почти градуированной структурой, обобщающей градуированную структуру классических аффинных алгебр. Напомним, что алгебра Ли $\mathscr{V}$ называется почти градуированной, если $\mathscr{V}=\bigoplus_{i} \mathscr{V}_{i}$, где $\operatorname{dim} \mathscr{V}_{i}<\infty,\left[\mathscr{V}_{i}, \mathscr{V}_{j}\right] \subseteq$ $\bigoplus_{k=i+j-k_{0}}^{i+j+k_{1}} \mathscr{N}_{k}$, а $k_{0}$ и $k_{1}$ не зависят от $i, j$.

Общее понятие почти градуированных алгебр и модулей над ними введено в [7]-[9], где были рассмотрены обобщения алгебр Гейзенберга и Вирасоро. Почти градуированная структура важна во многих вопросах, в том числе в теории представлений, аналогичных представлениям старшего веса (физически - во вторичном квантовании). В алгебрах Кричевера-Новикова почти градуированная структура задается с помощью базисов типа Фурье-Лорана на римановых поверхностях [7]. Она делает возможным построение конформной теории поля на пространстве модулей римановых поверхностей средствами алгебр Кричевера-Новикова [8]-[11].

Предметом настоящей работы являются центральные расширения. Описание центральных расширений является одним из фундаментальных вопросов теории когомологий алгебр Ли. Оно имеет большое значение для квантовой физики. Достаточно сказать, что алгебра Гейзенберга является одномерным центральным расширением коммутативной алгебры Ли.

Классы эквивалентности одномерных центральных расширений алгебры Ли $\mathscr{V}$ находятся во взаимно однозначном соответствии с элементами пространства $\mathrm{H}^{2}(\mathscr{V}, \mathbb{C})$ вторых когомологий алгебры Ли с коэффициентами в тривиальном модуле. В частности, такое центральное расширение явно задается 2-коциклом на $\mathscr{V}$. Если $\operatorname{dim} \mathrm{H}^{2}(\mathscr{V}, \mathbb{C})=1$, то существует только одно (с точностью до эквивалентности и умножения центрального элемента на число) нетривиальное центральное расширение. Допуская вольность, мы говорим в этом случае, что центральное расширение единственно.

История вопроса об описании центральных расширений алгебр токов на одномерных многообразиях началась с работ В. Каца [12] и Р. Муди [13], где было показано, что аффинные алгебры Ли, заданные каноническими образующими и соотношениями Картана-Серра, являются центральными расширениями алгебр петель. Г. Гарланд [14] доказал единственность одномерного центрального 
расширения для алгебр петель с простой алгеброй g. За дальнейшими комментариями по поводу центральных расширений алгебр петель мы отсылаем к $[15 ; \S 7.14]$.

Для более общего случая алгебр Ли вида $\mathfrak{g} \otimes \mathscr{A}$, где $\mathscr{A}$ - ассоциативная алгебра, а $\mathfrak{g}$ - простая алгебра Ли, К. Кассел [16] показал, что универсальное центральное расширение параметризуется кэлеровыми дифференциалами по модулю точных. В частности, оно не обязательно одномерно. Примером являются алгебры токов Кричевера-Новикова [17]-[20]. Они образованы $\mathfrak{g}$-значными мероморфными функциями на римановых поверхностях с полюсами лишь в точках фиксированного дивизора $D$. Для положительного рода или числа точек дивизора $D$ более двух одномерные центральные расширения существенно не единственны. Для простой алгебры $\mathfrak{g}$ они находятся во взаимно однозначном соответствии с элементами $\mathrm{H}_{1}(\Sigma \backslash \operatorname{supp}(D), \mathbb{R})$.

Единственность восстанавливается, если рассматривать центральные расширения в категории почти градуированных алгебр. Этот факт обнаружен И. М. Кричевером и С.П. Новиковым [7] в терминах введенных ими локальных коциклов. Коцикл $\gamma$ почти градуированной алгебры $\mathscr{V}$ называется локальным, если существует $K \in \mathbb{Z}$ такое, что $\gamma\left(\mathscr{V}_{i}, \mathscr{V}_{j}\right)=0$ для $|i+j|>K$. Центральное расширение является почти градуированным, если и только если задающий его коцикл локален. Для алгебр Кричевера-Новикова с простой алгеброй $\mathfrak{g}$ единственность почти градуированного центрального расширения доказана в [20]. Аналогичное утверждение было сделано для алгебр типа Вирасоро в [7], и был дан набросок его доказательства. Полная классификация почти градуированных центральных расширений для алгебр токов и векторных полей Кричевера-Новикова дана одним из авторов в [21], [20].

В настоящей статье мы решаем аналогичную задачу для алгебр операторов Лакса. Алгебры операторов Лакса дают общую точку зрения на постановку рассматриваемого вопроса и методы его решения. В этой связи отметим, что результат Кассела, существенно использовавшийся для алгебр Кричевера-Новикова, неприменим к алгебрам операторов Лакса, поскольку последние не расщепляются в тензорное произведение. Мы рассматриваем здесь только двухточечный случай, т. е. $D=P_{+}+P_{-}$. Принципиальная структура в многоточечном случае та же и будет рассмотрена в [22]. В этом случае снова оказывается, что если $\mathfrak{g}$ - классическая простая алгебра Ли, то $\overline{\mathfrak{g}}$ имеет единственное почти градуированное центральное расширение.

Опишем содержание статьи и полученные в ней результаты подробнее. Пусть $\mathscr{L}$ - алгебра Ли, состоящая из мероморфных векторных полей на $\Sigma$, голоморфных вне $\left\{P_{+}, P_{-}\right\}$. В разделе 2 мы вводим действие $\mathscr{L}$ на алгебре операторов Лакса $\overline{\mathfrak{g}}$. Для этого мы используем связности $\nabla^{(\omega)}$, введенные в [23]. Эти связности также имеют предписанное поведение в точках слабых особенностей и голоморфны вне их и $\left\{P_{+}, P_{-}\right\}$. На самом деле, мы могли бы даже потребовать (и делаем это), чтобы они были голоморфны в $P_{+}$. Алгебра операторов Лакса оказывается почти градуированным модулем над алгеброй мероморфных дифференциальных операторов степени не выше 1, голоморфных вне $\left\{P_{+}, P_{-}\right\}$. 
Структура $\mathscr{L}$-модуля, заданная выбором связности $\nabla^{(\omega)}$, позволяет нам ввести ниже важное понятие $\mathscr{L}$-инвариантного коцикла.

В разделе 3 мы рассматриваем следующие коциклы:

$$
\begin{aligned}
& \gamma_{1, \omega, C}\left(L, L^{\prime}\right)=\frac{1}{2 \pi \mathrm{i}} \int_{C} \operatorname{tr}\left(L \cdot \nabla^{(\omega)} L^{\prime}\right), \\
& \gamma_{2, \omega, C}\left(L, L^{\prime}\right)=\frac{1}{2 \pi \mathrm{i}} \int_{C} \operatorname{tr}(L) \cdot \operatorname{tr}\left(\nabla^{(\omega)} L^{\prime}\right),
\end{aligned}
$$

которые называем геометрическими коциклами. Здесь $C$ - произвольный цикл на $\Sigma$, не содержащий точек, в которых разрешены особенности. В другой форме коциклы вида (1.1) введены в [1]. Мы показываем, что соответствующие классы когомологий не зависят от выбора связности.

Коцикл $\gamma$ называется $\mathscr{L}$-инвариантным, если

$$
\gamma\left(\nabla_{e}^{(\omega)} L, L^{\prime}\right)+\gamma\left(L, \nabla_{e}^{(\omega)} L^{\prime}\right)=0
$$

для всех векторных полей $e \in \mathscr{L}$. Оказывается, что коциклы (1.1) и (1.2) $\mathscr{L}$-инвариантны. Мы называем класс когомологий $\mathscr{L}$-инвариантным, если он содержит $\mathscr{L}$-инвариантный коцикл, и локальным, если он содержит локальный коцикл. В случае простой алгебры Ли $\mathfrak{g}$ понятие $\mathscr{L}$-инвариантности позволяет нам выделить единственный элемент в классе когомологий. Более того, в случае $\overline{\mathfrak{g l}}(n)$ необходимо исключить нетривиальные коциклы, идущие от конечномерной алгебры Ли.

Помимо этих аспектов $\mathscr{L}$-инвариантность коцикла связана с тем, что он является ограничением коцикла на алгебре дифференциальных операторов, связанной с $\overline{\mathfrak{g}}$. Дальнейшую информацию по этому поводу см. в п. 3.3 .

Как и раньше, мы интересуемся только почти градуированными центральными расширениями, а следовательно, локальными коциклами (соответственно, классами когомологий). Для общего контура $C$ в (1.1) и (1.2) ни коцикл, ни его класс когомологий не являются локальными. Но если $C$ - контур, окружающий $P_{+}$, коцикл является таковым, см. предложение 3.7 .

Наш основной результат - теорема 3.9 , которая дает следующую классификацию. Для $\overline{\mathfrak{s l}}(n), \overline{\mathfrak{s o}}(n)$ и $\overline{\mathfrak{s p}}(2 n)$ пространство локальных классов когомологий одномерно. В каждом локальном классе когомологий имеется единственный $\mathscr{L}$-инвариантный коцикл. Он кратен коциклу (1.1) (где $C$ - контур, окружающий $\left.P_{+}\right)$. Для $\overline{\mathfrak{g l}}(n)$ пространство классов когомологий, которые локальны и, будучи ограничены на алгебру скалярных матриц, становятся $\mathscr{L}$-инвариантными, является двумерным. Более того, каждый локальный и $\mathscr{L}$-инвариантный коцикл является линейной комбинацией коциклов (1.1) и (1.2) (где $C$ - контур, окружающий $\left.P_{+}\right)$.

Доказательства содержатся в разделах 4 и 5. Мы следуем стратегии, разработанной в [21], и приспосабливаем ее к нашей более общей ситуации. В разделе 4 , используя локальность и $\mathscr{L}$-инвариантность, мы показываем, что каждый коцикл задается своими значениями на парах однородных элементов, для которых сумма степеней равна нулю. Более того, мы показываем, что 
$\mathscr{L}$-инвариантный и локальный коцикл однозначно определяется некоторым числом таких его значений. Более детальный анализ показывает, что такие коциклы имеют форму (1.1), (1.2).

В разделе 5 мы показываем следующее: пусть $\mathfrak{g}$ - простая конечномерная алгебра Ли и $\overline{\mathfrak{g}}-$ некоторая ассоциированная двухточечная алгебра токов, т. е. алгебра операторов Лакса, алгебра токов Кричевера-Новикова $\mathfrak{g} \otimes \mathscr{A}$ или Каца-Муди $\mathfrak{g} \otimes \mathbb{C}\left[z, z^{-1}\right]$. Тогда каждый локальный коцикл когомологичен коциклу, который вполне определен своими значениями на одной специальной паре элементов в $\overline{\mathfrak{g}}$ (именно, значением $\gamma\left(H_{1}^{\alpha}, H_{-1}^{\alpha}\right)$ для одного-единственного простого корня $\alpha$, см. обозначения в разделе 5). Следовательно, в этих случаях пространства когомологий самое большее одномерны. Комбинируя это с фактом существования коцикла (1.1), мы получаем существование и единственность локального класса когомологий. Далее, с точностью до умножения на число, (1.1) является единственным $\mathscr{L}$-инвариантным и локальным коциклом.

Мы существенно используем внутреннюю структуру алгебры Ли $\overline{\mathfrak{g}}$, связанную со структурой системы корней конечномерной простой алгебры Ли $\mathfrak{g}$, а также почти градуированность $\overline{\mathfrak{g}}$. Напомним, что в классическом случае $\mathfrak{g} \otimes \mathbb{C}\left[z, z^{-1}\right]$ алгебра градуирована. В этом очень специальном частном случае цепь наших аргументов становится проще и аналогична аргументации Гарланда [14].

Представленные аргументы останутся справедливыми и в более общем контексте, поскольку используются только внутренняя структура $\mathfrak{g}$, почти градуированность $\overline{\mathfrak{g}}$ и $\mathscr{L}$-инвариантность, см. замечание 5.18 в конце раздела 5.

Используя методы работы [20], можно получить соответствующие классификационные результаты для случая более чем двух точек, в которых разрешены "сильные" особенности. Точнее, пусть

$$
I:=\left\{P_{1}, P_{2}, \ldots, P_{K}\right\}, \quad O:=\left\{Q_{1}, Q_{2}, \ldots, Q_{L}\right\}
$$

- два непустых непересекающихся подмножества точек на $\Sigma$. Это те же предположения, что и для многоточечных алгебр Кричевера-Новикова, введенных и изучавшихся в [24]-[27], [21], [20]. Теперь в определении операторов Лакса мы разрешим им иметь полюсы в точках множества $I \cup O$. Расщепление на эти подмножества определяет почти градуировку алгебры. Можно показать, что в случае простой алгебры Ли пространство ограниченных сверху классов когомологий (т. е. таких, которые равны нулю на парах однородных элементов, сумма степеней которых превышает некий единый порог) является $K$-мерным $(K=\# I)$. В двухточечном случае всякий ограниченный коцикл является локальным. Для многих точек это не так. Техника, аналогичная предложенной в [20], дает единственность локального класса когомологий с точностью до пропорциональности. Соответствующий результат справедлив и для $\overline{\mathfrak{g l}}(n)$, а именно, пространство локальных и $\mathscr{L}$-инвариантных классов когомологий двумерно. Подробности будут приведены в готовящейся статье [22]. 


\section{2. Алгебры и структуры на них}

2.1. Алгебры. Пусть $\Sigma$ - компактная риманова поверхность рода $g$ с двумя отмеченными точками $P_{+}$и $P_{-}$. Возьмем $n \in \mathbb{N}$ и множество из $n g$ точек

$$
W:=\left\{\gamma_{s} \in \Sigma \backslash\left\{P_{+}, P_{-}\right\} \mid s=1, \ldots, n g\right\} .
$$

Каждой точке $\gamma_{s}$ сопоставим вектор $\alpha_{s} \in \mathbb{C}^{n}$. Система

$$
T:=\left\{\left(\gamma_{s}, \alpha_{s}\right) \in \Sigma \times \mathbb{C}^{n} \mid s=1, \ldots, n g\right\}
$$

называется ниже данными Тюрина. Эти данные связаны с модулями голоморфных векторных расслоений на $\Sigma$. В частности, для общих значений $\left(\gamma_{s}, \alpha_{s}\right)$ с $\alpha_{s} \neq 0$ наборы пар $\left(\gamma_{s},\left[\alpha_{s}\right]\right)$ с $\left[\alpha_{s}\right] \in \mathbb{C} P^{n-1}$ параметризуют полустабильные оснащенные голоморфные расслоения ранга $n$ и степени $n g$, см. [4].

Зафиксируем локальные координаты $z_{ \pm}$в окрестности $P_{ \pm}$и $z_{s}$ в окрестности $\gamma_{s}, s=1, \ldots, n g$. Пусть ниже $\mathfrak{g}$ - одна из матричных алгебр $\mathfrak{g l}(n), \mathfrak{s l}(n)$, $\mathfrak{s o}(n), \mathfrak{s p}(2 n)$ или $\mathfrak{s}(n)$, где последнее обозначает алгебру скалярных матриц.

Мы будем рассматривать мероморфные функции

$$
L: \Sigma \rightarrow \mathfrak{g}
$$

которые голоморфны вне $W \cup\left\{P_{+}, P_{-}\right\}$, имеют полюсы порядка не выше 1 (соответственно 2 для $\mathfrak{s p}(2 n))$ в точках множества $W$ и удовлетворяют некоторым условиям на $W$, зависящим от $T$ и $\mathfrak{g}$. Точки из $W$ мы называем слабыми особенностями. Эти объекты введены И. М. Кричевером для $\mathfrak{g l}(n)$ в контексте операторов Лакса на алгебраических кривых [2] и в дальнейшем обобщены в [1]. В частности, в последней работе возникли дополнительные требования к разложениям в точках $W$.

Для $\mathfrak{g l}(n)$ условия в точках множества $W$ таковы. Для всех $s=1, \ldots, n g$ функция $L$ имеет следующее разложение в $\gamma_{s} \in W$ :

$$
L\left(z_{s}\right)=\frac{L_{s,-1}}{z_{s}}+L_{s, 0}+\sum_{k>0} L_{s, k} z_{s}^{k} .
$$

Мы требуем, чтобы существовали $\beta_{s} \in \mathbb{C}^{n}$ и $\varkappa_{s} \in \mathbb{C}$ такие, что

$$
L_{s,-1}=\alpha_{s} \beta_{s}^{t}, \quad L_{s, 0} \alpha_{s}=\varkappa_{s} \alpha_{s}, \quad \operatorname{tr}\left(L_{s,-1}\right)=\beta_{s}^{t} \alpha_{s}=0 .
$$

Данные $T$ предполагаются фиксированными.

В частности, $L_{s,-1}$ - матрица ранга 1 , и если $\alpha_{s} \neq 0$, то $\alpha$ является собственным вектором $L_{s, 0}$. В [1] показано, что условия (2.5) не зависят от выбора координат $z_{s}$ и что совокупность таких функций образует ассоциативную алгебру относительно поточечного матричного умножения. Мы обозначим эту алгебру $\overline{\mathfrak{g l}}(n)$.

Несмотря на то, что алгебра $\overline{\mathfrak{g l}}(n)$ зависит от выбора как параметров Тюрина, так и точек $P_{+}$и $P_{-}$, мы опускаем эту зависимость в обозначениях. Заметим, что для $\lambda_{s} \in \mathbb{C}^{*}$ элементы $\alpha_{s}^{\prime}=\lambda_{s} \alpha_{s}$ и $\alpha_{s}$ при одном и том же $W$ определяют одну и ту же алгебру. 
Из ограничений (2.4) и (2.5) следует, что элементы алгебры операторов Лакса можно рассматривать как сечения расслоения эндоморфизмов $\operatorname{End}(B)$, где $B$ - векторное расслоение, соответствующее данным Тюрина.

Разложение в прямую сумму $\mathfrak{g l}(n)=\mathfrak{s}(n) \oplus \mathfrak{s l}(n)$, заданное соотношением

$$
X \mapsto\left(\frac{\operatorname{tr}(X)}{n} I_{n}, X-\frac{\operatorname{tr}(X)}{n} I_{n}\right),
$$

где $I_{n}-$ единичная матрица размера $n \times n$, индуцирует соответствующее расщепление алгебры операторов Лакса $\overline{\mathfrak{g l}}(n)$ :

$$
\overline{\mathfrak{g l}}(n)=\overline{\mathfrak{s}}(n) \oplus \overline{\mathfrak{s l}}(n) .
$$

Для $\overline{\mathfrak{s l}}(n)$ единственное дополнительное условие состоит в том, что в (2.4) все матрицы $L_{s, k}$ имеют нулевой след. Условие (2.5) остается неизменным.

Для $\overline{\mathfrak{s}}(n)$ все матрицы в $(2.4)$ скалярны. Поэтому соответствующий коэффициент разложения $L_{s,-1}$ для них обращается в 0 . В частности, элементы $\overline{\mathfrak{s}}(n)$ голоморфны в точках $W$. Кроме того, для $L_{s, 0}$, как для скалярной матрицы, каждый вектор $\alpha_{s}$ является собственным. Это означает, что кроме голоморфности других условий нет.

В случае $\mathfrak{s o}(n)$ мы требуем, чтобы все $L_{s, k}$ в (2.4) были кососимметричны. В частности, они являются бесследовыми. Следуя [1], мы требуем, чтобы параметры Тюрина $\alpha_{s}$ удовлетворяли соотношению $\alpha_{s}^{t} \alpha_{s}=0$. Далее, соотношения (2.5) приобретают следующую форму:

$$
L_{s,-1}=\alpha_{s} \beta_{s}^{t}-\beta_{s} \alpha_{s}^{t}, \quad \operatorname{tr}\left(L_{s,-1}\right)=\beta_{s}^{t} \alpha_{s}=0, \quad L_{s, 0} \alpha_{s}=\varkappa_{s} \alpha_{s} .
$$

Соотношения (2.8) не зависят от выбора координат $z_{s}$, и удовлетворяющие им отображения образуют алгебру Ли относительно поточечного матричного коммутирования [1].

Для $\mathfrak{s p}(2 n)$ мы рассмотрим симплектическую форму $\widehat{\sigma}$ на $\mathbb{C}^{2 n}$, заданную невырожденной кососимметрической матрицей $\sigma$. Не теряя общности, мы можем предположить, что эта матрица задана в стандартной форме $\sigma=\left(\begin{array}{cc}0 & I_{n} \\ -I_{n} & 0\end{array}\right)$. Алгебра Ли $\mathfrak{s p}(2 n)$ - это алгебра матриц $X$ таких, что $X^{t} \sigma+\sigma X=0$. Это эквивалентно соотношению $X^{t}=-\sigma X \sigma^{-1}$, из которого следует, что $\operatorname{tr}(X)=0$. Для стандартного вида $\sigma$ общий элемент алгебры $\mathfrak{s p}(2 n)$ имеет вид

$$
X=\left(\begin{array}{cc}
A & B \\
C & -A^{t}
\end{array}\right), \quad B^{t}=B, \quad C^{t}=C .
$$

В слабых особенностях мы имеем разложение

$$
L\left(z_{s}\right)=\frac{L_{s,-2}}{z_{s}^{2}}+\frac{L_{s,-1}}{z_{s}}+L_{s, 0}+L_{s, 1} z_{s}+\sum_{k>1} L_{s, k} z_{s}^{k} .
$$

Условие (2.5) модифицируется следующим образом [1]: существуют $\beta_{s} \in \mathbb{C}^{2 n}$, $\nu_{s}, \varkappa_{s} \in \mathbb{C}$ такие, что

$$
L_{s,-2}=\nu_{s} \alpha_{s} \alpha_{s}^{t} \sigma, \quad L_{s,-1}=\left(\alpha_{s} \beta_{s}^{t}+\beta_{s} \alpha_{s}^{t}\right) \sigma, \quad \beta_{s}^{t} \sigma \alpha_{s}=0, \quad L_{s, 0} \alpha_{s}=\varkappa_{s} \alpha_{s} .
$$


Более того, мы требуем выполнения равенства

$$
\alpha_{s}^{t} \sigma L_{s, 1} \alpha_{s}=0
$$

В [1] показано, что по отношению к поточечному матричному коммутатору совокупность отображений, удовлетворяющих этим условиям, образует алгебру Ли.

Суммируем вышесказанное в виде следующей теоремы.

Теорема 2.1 [1]. Пространство $\overline{\mathfrak{g}}$ операторов Лакса является алгеброй Ли по отношению $\kappa$ поточечному матричному коммутированию. Для $\overline{\mathfrak{g}}=\overline{\mathfrak{g l}}(n)$ оно является также ассочиативной алгеброй относительно поточечного матричного умножения.

Эти алгебры Ли называются алгебрами операторов Лакса.

Если мы возьмем в качестве параметров Тюрина $\alpha_{s}=0$, то слабых особенностей не будет. Таким образом получаются обычные двухточечные алгебры токов Кричевера-Новикова $\overline{\mathfrak{g}}=\mathfrak{g} \otimes \mathscr{A}$ [18]. Здесь $\mathscr{A}$ - алгебра мероморфных функций на $\Sigma$, голоморфных вне $P_{ \pm}$. С этой точки зрения алгебры операторов Лакса могут быть также названы обобщенными алгебрами Кричевера-Новикова.

Как отмечалось выше, для $\overline{\mathfrak{s}}(n)$ слабых особенностей нет, и нет условий на член нулевого порядка. Следовательно, $\overline{\mathfrak{s}}(n)$ совпадает с алгеброй функций Кричевера-Новикова, т. е.

$$
\overline{\mathfrak{s}}(n) \cong \mathfrak{s}(n) \otimes \mathscr{A} \cong \mathscr{A}
$$

как ассоциативные алгебры.

Заметим, что если в дополнение род римановой поверхности равен нулю, то алгебры операторов Лакса сводятся к алгебрам петель.

2.2. Почти градуированная структура. С помощью разложений в степенные ряды в точках $P_{+}$и $P_{-}$мы можем ввести почти градуировку, как это делалось для алгебр токов Кричевера-Новикова в [19], [20].

Чтобы выписать условия явно, мы должны ограничиться случаем, когда все отмеченные точки находятся в общем положении (включая точки из $W$ ). Пусть $\overline{\mathfrak{g}}$ - одна из алгебр операторов Лакса, введенных выше. Для $m \leqslant-g-1$ или $m \geqslant 1$ мы рассмотрим подпространство

$$
\begin{aligned}
\overline{\mathfrak{g}}_{m}:= & \left\{L \in \overline{\mathfrak{g}} \mid \exists X_{+}, X_{-} \in \mathfrak{g} \quad\right. \text { такие, что } \\
& \left.L\left(z_{+}\right)=X_{+} z_{+}^{m}+O\left(z_{+}^{m+1}\right), L\left(z_{-}\right)=X_{-} z_{-}^{-m-g}+O\left(z_{-}^{-m-g+1}\right)\right\} .
\end{aligned}
$$

Если $\mathfrak{g}$ полупроста и $\left\{\gamma_{s} \in W \mid \alpha_{s} \neq 0\right\} \neq \varnothing$, это определение остается в силе также для остальных значений $m$. Если $\mathfrak{g}$ равна $\mathfrak{g l}(n)$ или $\mathfrak{s}(n)$, то для $-g \leqslant$ $m \leqslant 0$ условия в $P_{-}$следует несколько изменить [1]. Фактически мы берем $\mathfrak{g l}(n)_{m}=\mathfrak{s l}(n)_{m} \oplus \mathfrak{s}(n)_{m}$ и используем для $\mathfrak{s l}(n)$ почти градуировку, введенную выше, а для $\mathfrak{s}(n) \cong \mathscr{A}$ - почти градуировку алгебры $\mathscr{A}$, которую мы напомним в п. 2.3, см. также [7]. Если $\left\{\gamma_{s} \in W \mid \alpha_{s} \neq 0\right\}=\varnothing$, то $\overline{\mathfrak{g}}=\mathfrak{g} \otimes \mathscr{A}$ и почти градуировка идет от $\mathscr{A}$, см. [19].

Назовем $\overline{\mathfrak{g}}_{m}$ однородным пространством степени $m$ в $\overline{\mathfrak{g}}$. 
ТеОрема 2.2 [1]. Алгебры Ли $\overline{\mathfrak{g}}$ являются почти градуированными по отношению $к$ только что введенной степени, более точно,

1) $\operatorname{dim} \overline{\mathfrak{g}}_{m}=\operatorname{dim} \mathfrak{g}$,

2) $\overline{\mathfrak{g}}=\bigoplus_{m \in \mathbb{Z}} \overline{\mathfrak{g}}_{m}$,

3) существует постоянное натуральное $M$ такое, что

$$
\left[\overline{\mathfrak{g}}_{m}, \overline{\mathfrak{g}}_{k}\right] \subseteq \bigoplus_{h=m+k}^{m+k+M} \overline{\mathfrak{g}}_{h}
$$

В [1] показано, что если $\mathfrak{g}=\mathfrak{s l}(n), \mathfrak{s p}(2 n), \mathfrak{s o}(n)$, то $M=g$. Нам в дальнейшем это не понадобится.

ЗАмЕчаниЕ 2.3. Результат о почти градуировке справедлив и тогда, когда точки $P_{+}, P_{-}$и элементы $W$ не находятся в общем положении. В этом случае порядок в точке $P_{-}$должен быть изменен.

ПреДЛОЖЕНИЕ 2.4. Пусть $X$ - элемент $\mathfrak{g}$. Для каждого $m$ существует единственный элемент $X_{m}$ в $\overline{\mathfrak{g}}_{m}$ такой, что

$$
X_{m}=X z_{+}^{m}+O\left(z_{+}^{m+1}\right) .
$$

ДокАЗАТЕЛЬСтво. Из первого утверждения теоремы 2.2 , т. е. из $\operatorname{dim} \overline{\mathfrak{g}}_{m}=$ $\operatorname{dim} \mathfrak{g}$, вытекает, что существует единственная комбинация базисных элементов такая, что выполняется (2.16).

Если дано $X \in \mathfrak{g}$, через $X_{m}$ обозначим единственный элемент в $\overline{\mathfrak{g}}_{m}$, определенный условием предложения 2.4.

Иногда будет полезно рассматривать также индуцированную фильтрацию

$$
F_{k}:=\bigoplus_{m \geqslant k} \overline{\mathfrak{g}}_{m}, \quad F_{k} \subseteq F_{k^{\prime}}, \quad k \geqslant k^{\prime}, \quad\left[F_{k}, F_{m}\right] \subseteq F_{k+m} .
$$

Результат (2.15) может быть дополнен следующим образом.

ПРЕДЛОЖЕНИЕ 2.5. Пусть $X_{k} \in \overline{\mathfrak{g}}_{k} u Y_{m} \in \overline{\mathfrak{g}}_{m}$ - элементы, ассоииированные с $X, Y \in \mathfrak{g}$ соответственно, тогда

$$
\left[X_{k}, Y_{m}\right]=[X, Y]_{k+m}+L
$$

где $[X, Y]-$ скобка в $\mathfrak{g} u L \in F_{k+m+1}$.

ДокАЗАТЕЛЬСТво. Используя для $X_{k}$ и $Y_{m}$ выражение $(2.16)$, получим

$$
\left[X_{k}, Y_{m}\right]=[X, Y] z_{+}^{k+m}+O\left(z_{+}^{k+m+1}\right) .
$$

Следовательно,

$$
\left[X_{k}, Y_{m}\right]-[X, Y]_{k+m}=O\left(z_{+}^{k+m+1}\right) \in F_{k+m+1},
$$

что и требовалось. 
Лемма 2.6. Пусть $\mathfrak{g}-$ простая $и$ y $\in \overline{\mathfrak{g}}$, тогда для каждого $m \in \mathbb{Z}$ существует конечное число элементов $y^{(i, 1)}, y^{(i, 2)} \in \overline{\mathfrak{g}}, i=1, \ldots, l=l(m)$, таких, что

$$
y-\sum_{i=1}^{l}\left[y^{(i, 1)}, y^{(i, 2)}\right] \in F_{m} .
$$

Доказательство. Если разложение $y$ в $P_{+}$начинается с порядка $k$, то $y=$ $X_{k}+y^{\prime}$, где $y^{\prime} \in F_{k+1}, X \in \mathfrak{g}$ и $X_{k}$ - соответствующий элемент степени $k$. Так как $\mathfrak{g}$ проста, то она совершенна, следовательно, существуют $X^{(1)}, X^{(2)} \in \mathfrak{g}$ такие, что $X=\left[X^{(1)}, X^{(2)}\right]$. Отсюда следует

$$
X_{k}=\left[X_{0}^{(1)}, X_{k}^{(2)}\right]+y^{\prime \prime}, \quad \text { где } y^{\prime \prime} \in F_{k+1}, \text { или } y=\left[X_{0}^{(1)}, X_{k}^{(2)}\right]+\left(y^{\prime}+y^{\prime \prime}\right) \text {. }
$$

Используя те же рассуждения для $\left(y+y^{\prime \prime}\right) \in F_{k+1}$, доказываем утверждение по индукции.

Алгебра Ли $\mathscr{V}$ называется совершенной, если $\mathscr{V}=[\mathscr{V}, \mathscr{V}]$. Алгебра операторов Лакса не является таковой. Лемму 2.6 можно рассматривать как более слабый аналог этого свойства. Заметим, что алгебры токов Кричевера-Новикова $\overline{\mathfrak{g}}$ для простых $\mathfrak{g}$ совершенны [20; предложение 3.2].

2.3. Структура модуля над $\mathscr{A}$ и $\mathscr{L}$. Здесь мы напомним определение алгебры функций Кричевера-Новикова $\mathscr{A}$ и векторных полей Кричевера-Новикова $\mathscr{L}$. Пусть $\mathscr{A}$ (соответственно $\mathscr{L})$ - пространство мероморфных функций (соответственно векторных полей) на $\Sigma$, голоморфных на $\Sigma \backslash\left\{P_{+}, P_{-}\right\}$. В частности, они также голоморфны в точках множества $W$. Очевидно, что $\mathscr{A}$ - ассоциативная алгебра относительно умножения функций, а $\mathscr{L}$ - алгебра Ли относительно коммутатора векторных полей. Путем указания специальных базисных элементов [7] эти алгебры наделяются почти градуированной структурой.

В случае $\mathscr{A}$ мы обозначаем указанный базис через $\left\{A_{m} \mid m \in \mathbb{Z}\right\}$. Элементы $A_{m}$ задаются условием $\operatorname{ord}_{P_{+}}\left(A_{m}\right)=m$ и дополнительным требованием в точке $P_{-}$, чтобы фиксировать $A_{m}$ однозначно с точностью до скалярного множителя. Для общего значения $m$ и общих точек $P_{+}$и $P_{-}$это требование таково: $\operatorname{ord}_{P_{-}}\left(A_{m}\right)=-m-g$. Чтобы зафиксировать скалярный множитель, мы требуем, чтобы локально в точке $P_{+}$, по отношению к выбранной локальной координате $z_{+}$, имело место разложение

$$
A_{m}\left(z_{+}\right)=z_{+}^{m}+O\left(z_{+}^{m+1}\right) .
$$

Мы полагаем $\mathscr{A}_{m}=\left\langle A_{m}\right\rangle$ и получаем структуру почти градуированной ассоциативной алгебры

$$
\mathscr{A}=\bigoplus_{m \in \mathbb{Z}} \mathscr{A}_{m}, \quad \mathscr{A}_{k} \cdot \mathscr{A}_{m} \subseteq \bigoplus_{h=k+m}^{k+m+M_{1}} \mathscr{A}_{h},
$$

с константой $M_{1}$, не зависящей от $k$ и $m$. Более того,

$$
A_{k} \cdot A_{m}=A_{k+m}+\sum_{h=k+m+1}^{k+m+M_{1}} \alpha_{k, m}^{h} A_{h}, \quad \alpha_{k, m}^{h} \in \mathbb{C} .
$$


Алгебра векторный полей $\mathscr{L}$ определяется аналогично. В этом случае базис - это $\left\{e_{m} \mid m \in \mathbb{Z}\right\}$ с условием $\operatorname{ord}_{P_{+}}\left(e_{m}\right)=m+1$, соответствующими порядками в $P_{-}$(в общем положении $\left.\operatorname{ord}_{P_{-}}\left(e_{m}\right)=-m-3 g-3\right)$ и локальным разложением в $P_{+}$

$$
e_{m}\left(z_{+}\right)=\left(z_{+}^{m+1}+O\left(z_{+}^{m+2}\right)\right) \frac{d}{d z_{+}} .
$$

Мы полагаем $\mathscr{L}_{m}=\left\langle e_{m}\right\rangle$ и получаем почти градуированную структуру

$$
\mathscr{L}=\bigoplus_{m \in \mathbb{Z}} \mathscr{L}_{m}, \quad\left[\mathscr{L}_{k}, \mathscr{L}_{m}\right] \subseteq \bigoplus_{h=k+m}^{k+m+M_{2}} \mathscr{L}_{h}
$$

с константой $M_{2}$, не зависящей от $k$ и $m$. Для базисных элементов имеем

$$
\left[e_{k}, e_{m}\right]=(m-k) e_{k+m}+\sum_{h=k+m+1}^{k+m+M_{2}} \beta_{k, m}^{h} e_{h}, \quad \beta_{k, m}^{h} \in \mathbb{C} .
$$

Элементы алгебры Ли $\mathscr{L}$ действуют на $\mathscr{A}$ дифференцированиями. Это превращает пространство $\mathscr{A}$ в почти градуированный модуль над $\mathscr{L}$. В частности, мы имеем

$$
e_{k} \cdot A_{m}=m A_{k+m}+\sum_{h=k+m+1}^{k+m+M_{3}} \epsilon_{k, m}^{h} A_{h}, \quad \epsilon_{k, m}^{h} \in \mathbb{C},
$$

с константой $M_{3}$, не зависящей от $k$ и $m$. Константы $M_{i}$ можно легко вычислить [7], но их точные значения не играют роли в дальнейшем.

Пространство $\overline{\mathfrak{g}}$ является модулем над $\mathscr{A}$ относительно поточечного умножения. Очевидно, соотношения (2.4), (2.5), (2.8), (2.11) не нарушаются. Прямой подсчет порядков в точках $P_{+}$и $P_{-}$показывает, что существует константа $M_{4}$ (не зависящая от $k$ и $m$ ) такая, что

$$
\mathscr{A}_{k} \cdot \overline{\mathfrak{g}}_{m} \subseteq \bigoplus_{h=k+m}^{k+m+M_{4}} \overline{\mathfrak{g}}_{h}
$$

Рассматривая степень в $P_{+}$, мы видим, что для $X \in \mathfrak{g}$

$$
A_{m} \cdot X_{0}=X_{m}+L, \quad L \in F_{m+1}
$$

Вообще говоря, неверно, что $A_{m} \cdot X_{0}=X_{m}$, поскольку порядки в $P_{-}$не совпадают. Также, так как $\alpha \neq 0$, то элемент $A_{m} \cdot X$ не обязательно принадлежит $\overline{\mathfrak{g}}$, поскольку $\alpha$ не обязательно является собственным вектором $X$. Заметим, что $A_{m} \cdot X$ всегда является элементом алгебры токов Кричевера-Новикова $\mathfrak{g} \otimes \mathscr{A}$.

Теперь введем действие $\mathscr{L}$ на $\overline{\mathfrak{g}}$. Напомним, что $\overline{\mathfrak{g}}=\overline{\mathfrak{g l}}(n)$ следует интерпретировать как алгебру эндоморфизмов пространства мероморфных сечений векторного расслоения. Действие $\mathscr{L}$ на $\overline{\mathfrak{g}}$ должно определяться некоторой связностью на этих сечениях. Обозначим эту связность $\nabla^{(\omega)}$, где $\omega$ - форма связности.

Мы вводим связность $\nabla^{(\omega)}$, следуя работам [2], [23], с некоторыми изменениями. Форма связности $\omega$ мероморфна, голоморфна вне $P_{+}, P_{-}$и $W$, имеет 
предписанное поведение в точках множества $W$ и принимает теперь значения в g. Если $s$ таково, что $\alpha_{s}=0$, мы требуем, чтобы $\omega$ также была регулярна в $\gamma_{s}$. Для точек $\gamma_{s}$ с $\alpha_{s} \neq 0$ мы требуем, чтобы имело место разложение

$$
\omega\left(z_{s}\right)=\left(\frac{\omega_{s,-1}}{z_{s}}+\omega_{s, 0}+\sum_{k \geqslant 1} \omega_{s, k} z_{s}^{k}\right) d z_{s} .
$$

Следующие условия были даны в [2] для $\mathfrak{g l}(n)$ и в [1] для других классических алгебр Ли. Для $\mathfrak{g l}(n)$ предполагается, что существуют $\widetilde{\beta}_{s} \in \mathbb{C}^{n}$ и $\widetilde{\varkappa}_{s} \in \mathbb{C}$ такие, что

$$
\omega_{s,-1}=\alpha_{s} \widetilde{\beta}_{s}^{t}, \quad \omega_{s, 0} \alpha_{s}=\widetilde{\varkappa}_{s} \alpha_{s}, \quad \operatorname{tr}\left(\omega_{s,-1}\right)=\widetilde{\beta}_{s}^{t} \alpha_{s}=1 .
$$

Заметим, что по сравнению с (2.5) было изменено только последнее условие. Для $\mathfrak{s o}(n)$ предполагается, что существуют $\widetilde{\beta}_{s} \in \mathbb{C}^{n}$ и $\widetilde{\varkappa}_{s} \in \mathbb{C}$ такие, что

$$
\omega_{s,-1}=\alpha_{s} \widetilde{\beta}_{s}^{t}-\widetilde{\beta}_{s} \alpha_{s}^{t}, \quad \omega_{s, 0} \alpha_{s}=\widetilde{\varkappa}_{s} \alpha_{s}, \quad \widetilde{\beta}_{s}^{t} \alpha_{s}=1 .
$$

Для $\mathfrak{s p}(2 n)$ предполагается, что существуют $\widetilde{\beta}_{s} \in \mathbb{C}^{2 n}$ и $\widetilde{\varkappa}_{s} \in \mathbb{C}$ такие, что

$$
\omega_{s,-1}=\left(\alpha_{s} \widetilde{\beta}_{s}^{t}+\widetilde{\beta}_{s} \alpha_{s}^{t}\right) \sigma, \quad \omega_{s, 0} \alpha_{s}=\widetilde{\varkappa}_{s} \alpha_{s}, \quad \alpha_{s}^{t} \sigma \omega_{s, 1} \alpha_{s}=0, \quad \widetilde{\beta}_{s}^{t} \sigma \alpha_{s}=1 .
$$

ЗАмечАние 2.7. По сравнению с (2.5), (2.8), (2.11) условие $\beta_{s}^{t} \alpha^{s}=0$ (соответственно $\beta_{s}^{t} \sigma \alpha^{s}=0$ ) заменено условием $\widetilde{\beta}_{s}^{t} \alpha^{s}=1$ (соответственно условием $\left.\widetilde{\beta}_{s}^{t} \sigma \alpha^{s}=1\right)$. Для $\mathfrak{s p}(2 n)$ мы могли бы также рассматривать в точках $\gamma_{s}$ полюсы порядка два с главным членом вида $\left(\widetilde{\nu} \alpha_{s} \alpha_{s}^{t} \sigma\right) / z_{s}^{2}$ без всяких изменений в дальнейшем.

Существование нетривиальных связностей, удовлетворяющих перечисленным условиям, доказывается точно так же, как п. 1) теоремы 2.2, т. е. в основном с помощью теоремы Римана-Роха. Мы можем даже потребовать, и в дальнейшем всегда делаем это, чтобы форма связности была голоморфна в $P_{+}$. Заметим, что если все $\alpha_{s}=0$, мы могли бы взять $\omega=0$.

Связность $\nabla^{(\omega)}$ индуцирует следующую связность на $\overline{\mathfrak{g}}$, которую мы тоже обозначаем $\nabla^{(\omega)}$ :

$$
\nabla^{(\omega)}=d+[\omega, \cdot]
$$

Если форма $\omega$ фиксирована, мы обычно не упоминаем ее в обозначениях. Пусть $e$ - векторное поле. В локальных координатах форма связности и векторное поле представляются как $\omega=\widetilde{\omega} d z$ и $e=\tilde{e} \frac{d}{d z}$, где $\tilde{e}-$ локальная функция, а $\widetilde{\omega}-$ локальная матричнозначная функция. Ковариантная производная в направлении е дается соотношением

$$
\nabla_{e}^{(\omega)}=d z(e) \frac{d}{d z}+[\omega(e), \cdot]=e \cdot+[\widetilde{\omega} \tilde{e}, \cdot]=\tilde{e} \cdot\left(\frac{d}{d z}+[\widetilde{\omega}, \cdot]\right) .
$$

Здесь первый член соответствует взятию обычной производной в каждом матричном элементе.

Используя последнее описание, мы можем легко проверить, что для $L \in \overline{\mathfrak{g}}$, $g \in \mathscr{A}, e, f \in \mathscr{L}$ выполнены равенства

$$
\nabla_{e}^{(\omega)}(g \cdot L)=(e . g) \cdot L+g \cdot \nabla_{e}^{(\omega)} L, \quad \nabla_{g \cdot e}^{(\omega)} L=g \cdot \nabla_{e}^{(\omega)} L
$$


и

$$
\nabla_{[e, f]}^{(\omega)}=\left[\nabla_{e}^{(\omega)}, \nabla_{f}^{(\omega)}\right] .
$$

ПрЕДЛОЖЕНИЕ 2.8. $\nabla_{e}^{(\omega)}$ действует как дифберенцирование алгебры Ли $\overline{\mathfrak{g}}$, m.e.

$$
\nabla_{e}^{(\omega)}\left[L, L^{\prime}\right]=\left[\nabla_{e}^{(\omega)} L, L^{\prime}\right]+\left[L, \nabla_{e}^{(\omega)} L^{\prime}\right] .
$$

ДокАзАтельство. Сначала заметим, что локальная функция $\tilde{e}$ коммутирует со всеми матрицами. Далее,

$$
\begin{aligned}
\nabla_{e}^{(\omega)}\left[L, L^{\prime}\right] & =\tilde{e} \cdot\left(\frac{d\left[L, L^{\prime}\right]}{d z}+\left[\widetilde{\omega},\left[L, L^{\prime}\right]\right]\right) \\
& =\tilde{e} \cdot\left(\left[\frac{d L}{d z}, L^{\prime}\right]+\left[L, \frac{d L^{\prime}}{d z}\right]+\left[\widetilde{\omega},\left[L, L^{\prime}\right]\right]\right) \\
{\left[\nabla_{e}^{(\omega)} L, L^{\prime}\right] } & =\tilde{e} \cdot\left(\left[\frac{d L}{d z}, L^{\prime}\right]+\left[[\widetilde{\omega}, L], L^{\prime}\right]\right) \\
{\left[L, \nabla_{e}^{(\omega)} L^{\prime}\right] } & =\tilde{e} \cdot\left(\left[L, \frac{d L^{\prime}}{d z}\right]+\left[L,\left[\widetilde{\omega}, L^{\prime}\right]\right]\right)
\end{aligned}
$$

Соотношение (2.38) следует из тождества Якоби для коммутатора матриц.

ПРедлОжЕНИЕ 2.9. Ковариантное дифберенцирование превращает $\overline{\mathfrak{g}}$ в модуль над алгеброй Ли $\mathscr{L}$.

ДокАЗАТЕЛьСтво. Поскольку форма связности принимает значения в $\mathfrak{g}$, для $L \in \overline{\mathfrak{g}}$ ковариантная производная $\nabla_{e}^{(\omega)} L$ является $\mathfrak{g}$-значной мероморфной функцией. Очевидно, не появляется никаких новых полюсов. Мы должны проверить, что поведение в точках слабых особенностей является предписанным. В частности, нам надо проверить, что там нет полюсов порядка два и более (порядка $\geqslant 3$ для $\overline{\mathfrak{s p}}(2 n))$. Из (2.37) следует, что $\overline{\mathfrak{g}}$ будет модулем над алгеброй Ли $\mathscr{L}$. Здесь мы рассмотрим только случай $\mathfrak{g l}(n)$. Доказательство для $\mathfrak{s o}(n)$ и $\mathfrak{s p}(2 n)$ аналогично, но требует больших вычислений, поэтому случаи $\mathfrak{s o}(n)$ и $\mathfrak{s p}(2 n)$ рассматриваются в приложении А.

Пусть $\gamma_{s}$ - точка $W$. Если $\alpha_{s}=0$, то операторы Лакса не имеют полюса в $\gamma_{s}$ и свободны от связанных с ним условий на члены разложений нулевого и первого порядков. По условию, наша форма связности голоморфна в $\gamma_{s}$, и, значит, $\nabla_{e}^{(\omega)} L$ имеет правильное поведение в $\gamma_{s}$. Следовательно, единственный случай, который надо проверять, - это $\alpha_{s} \neq 0$. Для простоты мы опустим индекс $s$. В частности, $z$ будет обозначать $z_{s}$. Так как значение $\tilde{e}$ в точке $\gamma_{s}$ является скаляром, мы можем игнорировать его в вычислениях. Мы также используем одно и то же обозначение для формы $\omega$ и представляющей ее матричной функции. Мы берем разложения, удовлетворяющие условиям (2.5) и (2.31) соответственно:

$$
L(z)=\frac{L_{-1}}{z}+L_{0}+L_{1} z+O\left(z^{2}\right), \quad \omega(z)=\frac{\omega_{-1}}{z}+\omega_{0}+\omega_{1} z+O\left(z^{2}\right) .
$$

Тогда

$$
\frac{d L}{d z}(z)=\frac{-L_{-1}}{z^{2}}+L_{1}+O\left(z^{1}\right)
$$




$$
\begin{gathered}
{[\omega, L]=\frac{1}{z^{2}}\left[\omega_{-1}, L_{-1}\right]+\frac{1}{z}\left(\left[\omega_{-1}, L_{0}\right]+\left[\omega_{0}, L_{-1}\right]\right)} \\
+\left(\left[\omega_{-1}, L_{1}\right]+\left[\omega_{0}, L_{0}\right]+\left[\omega_{1}, L_{-1}\right]\right)
\end{gathered}
$$

Для полюса порядка два мы вычисляем матричный коэффициент следующим образом:

$$
-L_{-1}+\left[\omega_{-1}, L_{-1}\right]=-\alpha \beta^{t}+\left[\alpha \widetilde{\beta}^{t}, \alpha \beta^{t}\right]=-\alpha \beta^{t}+\alpha \widetilde{\beta}^{t} \alpha \beta^{t}-\alpha \beta^{t} \alpha \widetilde{\beta}^{t}=0 .
$$

Здесь мы воспользовались тем, что $\widetilde{\beta}^{t} \alpha=1$ и $\beta^{t} \alpha=0$.

Матричный коэффициент члена первого порядка равен

$$
\begin{aligned}
{\left[\omega_{-1}, L_{0}\right]+\left[\omega_{0}, L_{-1}\right] } & =\alpha \widetilde{\beta}^{t} L_{0}-L_{0} \alpha \widetilde{\beta}^{t}-\omega_{0} \alpha \beta^{t}+\alpha \beta^{t} \omega_{0} \\
& =\alpha\left(\widetilde{\beta}^{t} L_{0}-\varkappa \widetilde{\beta}^{t}-\tilde{\varkappa} \beta^{t}+\beta^{t} \omega_{0}\right)=\alpha \widehat{\beta}^{t}
\end{aligned}
$$

где $\widehat{\beta}^{t}$ обозначает множитель в скобках. Вычисляя, получаем

$$
\widehat{\beta}^{t} \alpha=\left(\widetilde{\beta}^{t} L_{0}-\varkappa \widetilde{\beta}^{t}-\tilde{\varkappa} \beta^{t}+\beta^{t} \omega_{0}\right) \alpha=\varkappa \widetilde{\beta}^{t} \alpha-\varkappa \widetilde{\beta}^{t} \alpha-\tilde{\varkappa} \beta^{t} \alpha+\tilde{\varkappa} \beta^{t} \alpha=0 .
$$

Здесь мы несколько раз использовали соотношения $L_{0} \alpha=\varkappa \alpha$ и $\omega_{0} \alpha=\tilde{\varkappa} \alpha$.

Наконец, мы должны показать, что $\alpha$ является собственным значением члена нулевой степени, т. е. что вектор

$$
L_{1} \alpha+\left[\omega_{-1}, L_{1}\right] \alpha+\left[\omega_{0}, L_{0}\right] \alpha+\left[\omega_{1}, L_{-1}\right] \alpha
$$

кратен $\alpha$. Заметим, что $\left[\omega_{0}, L_{0}\right] \alpha=0$, так как $\alpha$ - собственный вектор обеих матриц. Для оставшихся слагаемых имеем

$$
L_{1} \alpha+\alpha \widetilde{\beta}^{t} L_{1} \alpha-L_{1} \alpha \widetilde{\beta}^{t} \alpha+\omega_{1} \alpha \beta^{t} \alpha-\alpha \beta^{t} \omega_{1} \alpha=\alpha\left(\widetilde{\beta}^{t} L_{1} \alpha-\beta^{t} \omega_{1} \alpha\right) .
$$

Заметим, что второй сомножитель скалярен. Отсюда и вытекает доказываемое.

ПРЕДЛОЖЕНИЕ 2.10. Разложение $\overline{\mathfrak{g l}}(n)=\overline{\mathfrak{s}}(n) \oplus \overline{\mathfrak{s l}}(n)-$ это разложение $\mathscr{L}$-модулей, т.е.

$$
\nabla_{e}: \overline{\mathfrak{s}}(n) \rightarrow \overline{\mathfrak{s}}(n), \quad \nabla_{e}: \overline{\mathfrak{s l}}(n) \rightarrow \overline{\mathfrak{s l}}(n)
$$

Более того, ввиду отождествления (2.13) $\mathscr{L}$-модуль $\overline{\mathfrak{s}}(n)$ эквивалентен $\mathscr{L}$-модулю $\mathscr{A}$.

ДокАЗАТЕЛЬство. Ввиду (2.35) связность, примененная к бесследовым матрицам, дает снова бесследовую матрицу (коммутатор всегда имеет нулевой след), т. е. результат лежит в $\overline{\mathfrak{s l}}(n)$. Для скалярных матриц коммутатор вообще обращается в нуль. Следовательно, $\nabla_{e}$ не зависит от формы связности и остается только обычное действие $\mathscr{L}$ на $\mathscr{A}$.

ПрЕДЛОЖЕНИЕ 2.11. (а) $\overline{\mathfrak{g}}$ является почти градуированным $\mathscr{L}$-модулем.

(b) Главный член разложения дается соотношением

$$
\nabla_{e_{k}} X_{m}=m X_{k+m}+L, \quad L \in F_{k+m+1} .
$$


ДокАЗАТЕЛьСтво. (а) Применим (2.35) к однородным элементам:

$$
\nabla_{e_{k}} X_{m}=e_{k} \cdot X_{m}+\left[\widetilde{\omega} \tilde{e}_{k}, X_{m}\right]
$$

Форма $\omega$ имеет фиксированные порядки в $P_{+}$и $P_{-}$, действие $\mathscr{L}$ на $\mathscr{A}$ почти градуированно, и скобка соответствует коммутатору в почти градуированной алгебре $\overline{\mathfrak{g}}$. Все вместе дает доказываемое.

(b) В окрестности $P_{+}$

$$
X_{m}=X z_{+}^{m}+O\left(z_{+}^{m+1}\right), \quad e_{k}=z_{+}^{k+1} \frac{d}{d z}+O\left(z_{+}^{k+2}\right) .
$$

Отсюда следует

$$
e_{k} \cdot X_{m}=m X z_{+}^{k+m}+O\left(z_{+}^{k+m+1}\right), \quad \widetilde{\omega} \tilde{e}_{k}=B z_{+}^{k+1}+O\left(z_{+}^{k+2}\right),
$$

где $B \in \mathfrak{g l}(n)$. Следовательно,

$$
\left[\widetilde{\omega} \tilde{e}_{k}, X_{m}\right]=O\left(z^{k+m+1}\right)
$$

и (2.48) вытекает из (2.49).

Если $\omega$ имеет в $P_{+}$полюс порядка 1 , то степень главного члена разложения все еще будет равна $k+m$, но коэффициент будет другим.

2.4. Структура модуля над $\mathscr{D}^{1}$ и $\mathscr{D}_{\mathfrak{g}}^{1}$. Алгебра Ли $\mathscr{D}^{1}$ мероморфных дифференциальных операторов на $\Sigma$ степени не выше 1 , голоморфных вне $\left\{P_{+}, P_{-}\right\}$, определяется как полупрямая сумма $\mathscr{A}$ и $\mathscr{L}$ с коммутатором между ними, данным действием $\mathscr{L}$ на $A$. Как векторное пространство $\mathscr{D}^{1}$ расщепляется в прямую сумму $\mathscr{D}^{1}=\mathscr{A} \oplus \mathscr{L}$ и скобка задается соотношением

$$
[(g, e),(h, f)]:=(e . h-f \cdot g,[e, f]) .
$$

В частности,

$$
[e, h]=e . h .
$$

Это почти градуированная алгебра Ли [21].

ПРЕДЛОЖениЕ 2.12. Алгебры операторов Лакса $\overline{\mathfrak{g}}$ являются почти градуированными модулями над $\mathscr{D}^{1}$ с действием

$$
\text { e.L }:=\nabla_{e}^{(\omega)} L, \quad h . L:=h \cdot L .
$$

ДоказАТЕЛЬство. Так как $\overline{\mathfrak{g}}$ является почти градуированным $\mathscr{A}$ - и $\mathscr{L}$-модулем, достаточно показать, что выполнено соотношение (2.54). Для $e \in \mathscr{L}$, $h \in \mathscr{A}, L \in \overline{\mathfrak{g}}$, используя (2.35), получаем

$$
\begin{aligned}
& \text { e.(h.L) }-h .(e . L)=\nabla_{e}^{(\omega)}(h L)-h \nabla_{e}^{(\omega)}(L) \\
& \qquad \tilde{e}\left(\frac{d(h L)}{d z}+[\widetilde{\omega}, h L]\right)-h \tilde{e}\left(\frac{d L}{d z}+[\widetilde{\omega}, L]\right)=\left(\tilde{e} \frac{d h}{d z}\right) L=(e . h) L=[e, h] . L .
\end{aligned}
$$

Предложение 2.12 доказано. 
В этой связи возникает еще одна структура. Алгебра операторов Лакса $\overline{\mathfrak{g}}$ является модулем над алгеброй Ли $\mathscr{L}$, которая действует на ней дифференцированиями (согласно предложению 2.8). Следовательно, как и выше, мы можем рассмотреть полупрямую сумму $\mathscr{D}_{\mathfrak{g}}^{1}=\overline{\mathfrak{g}} \oplus \mathscr{L}$ со скобкой Ли, заданной для смешанных пар соотношением

$$
[e, L]:=e \cdot L=\nabla_{e}^{(\omega)} L
$$

(см. в [20] соответствующую конструкцию для алгебр Кричевера-Новикова аффинного типа; аналогично [20] могут быть изучены и классифицированы центральные расширения алгебры $\mathscr{D}_{\mathfrak{g}}^{1}$; подробности будут опубликованы в другом месте).

\section{3. Коциклы}

3.1. Геометрические коциклы. В этом пункте мы вводим геометрические 2-коциклы на алгебре Ли $\overline{\mathfrak{g}}$ со значениями в $\mathbb{C}$. Соответствующее пространство когомологий $\mathrm{H}^{2}(\overline{\mathfrak{g}}, \mathbb{C})$ классифицирует классы эквивалентности (одномерных) центральных расширений алгебры $\overline{\mathfrak{g}}$.

Напомним, что 2-коцикл на $\overline{\mathfrak{g}}-$ это билинейная кососимметрическая форма $\gamma: \overline{\mathfrak{g}} \times \overline{\mathfrak{g}} \rightarrow \mathbb{C}$, которая удовлетворяет условию

$$
\gamma\left(\left[L, L^{\prime}\right], L^{\prime \prime}\right)+\gamma\left(\left[L^{\prime}, L^{\prime \prime}\right], L\right)+\gamma\left(\left[L^{\prime \prime}, L\right], L^{\prime}\right)=0 .
$$

2 -коцикл $\gamma$ является кограницей, если существует линейная форма $\phi$ на $\overline{\mathfrak{g}}$ такая, что

$$
\gamma\left(L, L^{\prime}\right)=\phi\left(\left[L, L^{\prime}\right]\right), \quad L, L^{\prime} \in \overline{\mathfrak{g}} .
$$

Связь коциклов с центральными расширениями алгебры $\overline{\mathfrak{g}}$ такова. Пусть дан 2-коцикл $\gamma$ алгебры $\overline{\mathfrak{g}}$, тогда соответствующее центральное расширение $\hat{\mathfrak{g}}_{\gamma}$ задается как прямая сумма векторных пространств $\hat{\mathfrak{g}}_{\gamma}=\overline{\mathfrak{g}} \oplus \mathbb{C} \cdot t$ со скобкой Ли

$$
\left[\widehat{L}, \widehat{L^{\prime}}\right]=\widehat{\left[L, L^{\prime}\right]}+\gamma\left(L, L^{\prime}\right) \cdot t, \quad[\widehat{L}, t]=0, \quad L, L^{\prime} \in \overline{\mathfrak{g}} .
$$

Здесь мы использовали $\widehat{L}:=(L, 0)$ и $t:=(0,1)$. Обратно, каждое центральное расширение

$$
0 \longrightarrow \mathbb{C} \longrightarrow \hat{\mathfrak{g}} \longrightarrow \overline{\mathfrak{g}} \longrightarrow 0
$$

определяет 2-коцикл $\gamma: \overline{\mathfrak{g}} \rightarrow \mathbb{C}$, для чего надо выбрать сечение $s: \overline{\mathfrak{g}} \rightarrow \hat{\mathfrak{g}}$.

Два центральных расширения $\hat{\mathfrak{g}}_{\gamma}$ и $\hat{\mathfrak{g}}_{\gamma^{\prime}}$ эквивалентны, если определяющие их коциклы $\gamma$ и $\gamma^{\prime}$ когомологичны.

Пусть $\omega$ - форма связности, введенная в предыдущем разделе при определении связности (2.34). Далее, пусть $C$ - гладкий контур на $\Sigma$, не содержащий точек из $\left\{P_{+}, P_{-}\right\} \cup W$. Определим следующие билинейные формы на $\overline{\mathfrak{g}}$ :

$$
\begin{array}{ll}
\gamma_{1, \omega, C}\left(L, L^{\prime}\right)=\frac{1}{2 \pi \mathrm{i}} \int_{C} \operatorname{tr}\left(L \cdot \nabla^{(\omega)} L^{\prime}\right), & L, L^{\prime} \in \overline{\mathfrak{g}}, \\
\gamma_{2, \omega, C}\left(L, L^{\prime}\right)=\frac{1}{2 \pi \mathrm{i}} \int_{C} \operatorname{tr}(L) \cdot \operatorname{tr}\left(\nabla^{(\omega)} L^{\prime}\right), & L, L^{\prime} \in \overline{\mathfrak{g}} .
\end{array}
$$


ПРЕДЛОЖЕНИЕ 3.1. Билинейные формы $\gamma_{1, \omega, C} u \gamma_{2, \omega, C}$ являются коииклами.

ДокАЗАтельство. Начнем с $\gamma_{2, \omega, C}$. Для подынтегральной формы находим

$$
\operatorname{tr}(L) \cdot \operatorname{tr}\left(\nabla^{(\omega)} L^{\prime}\right)=\operatorname{tr}(L) \cdot \operatorname{tr}\left(d L^{\prime}+\left[\omega, L^{\prime}\right]\right)=\operatorname{tr}(L) \cdot \operatorname{tr}\left(d L^{\prime}\right) .
$$

Далее, $h:=\operatorname{tr}(L) \cdot \operatorname{tr}\left(L^{\prime}\right)$ является мероморфной функцией и

$$
d h=d\left(\operatorname{tr}(L) \cdot \operatorname{tr}\left(L^{\prime}\right)\right)=(d(\operatorname{tr}(L))) \cdot \operatorname{tr}\left(L^{\prime}\right)+\operatorname{tr}(L) \cdot d\left(\operatorname{tr}\left(L^{\prime}\right)\right) .
$$

По теореме Стокса $\frac{1}{2 \pi \mathrm{i}} \int_{C} d h=0$ и, следовательно,

$$
\frac{1}{2 \pi \mathrm{i}} \int_{C} \operatorname{tr}(L) \operatorname{tr}\left(d L^{\prime}\right)=-\frac{1}{2 \pi \mathrm{i}} \int_{C} \operatorname{tr}\left(L^{\prime}\right) \operatorname{tr}(d L),
$$

что означает антисимметрию. Очевидно, $\gamma_{2, \omega, C}\left(\left[L, L^{\prime}\right], L^{\prime \prime}\right)=0$ и условие $(3.1)$ выполнено.

Далее мы рассмотрим $\gamma_{1, \omega, C}$ и запишем в локальных координатах $\omega=\widetilde{\omega} d z$. Подынтегральная форма может быть записана как

$$
\operatorname{tr}\left(L \cdot \nabla^{(\omega)} L^{\prime}\right)=\operatorname{tr}\left(L \cdot\left(d L^{\prime}+\left[\widetilde{\omega}, L^{\prime}\right] d z\right)\right)=\operatorname{tr}\left(L \cdot d L^{\prime}\right)+\operatorname{tr}\left(L \cdot\left[\widetilde{\omega}, L^{\prime}\right]\right) d z .
$$

Положим $h=\operatorname{tr}\left(L \cdot L^{\prime}\right)$, тогда

$$
d h=d\left(\operatorname{tr}\left(L \cdot L^{\prime}\right)\right)=\operatorname{tr}\left(d L \cdot L^{\prime}\right)+\operatorname{tr}\left(L \cdot d L^{\prime}\right)=\operatorname{tr}\left(L^{\prime} \cdot d L\right)+\operatorname{tr}\left(L \cdot d L^{\prime}\right) .
$$

По теореме Стокса интеграл $d h$ по $C$ обращается в нуль, следовательно, первый член в (3.7) кососимметричен. Для второго члена находим

$$
\operatorname{tr}\left(L \cdot\left[\widetilde{\omega}, L^{\prime}\right]\right)=\operatorname{tr}\left(L \cdot \widetilde{\omega} \cdot L^{\prime}-L \cdot L^{\prime} \cdot \widetilde{\omega}\right)=\operatorname{tr}\left(L^{\prime} \cdot L \cdot \widetilde{\omega}-L^{\prime} \cdot \widetilde{\omega} \cdot L\right)=-\operatorname{tr}\left(L^{\prime} \cdot[\widetilde{\omega}, L]\right) .
$$

Следовательно, второй член также антисимметричен. Для проверки условия (3.1) запишем

$$
\operatorname{tr}\left(\left[L, L^{\prime}\right] \cdot \nabla^{(\omega)} L^{\prime \prime}\right)=\operatorname{tr}\left(\left[L, L^{\prime}\right] \cdot d L^{\prime \prime}\right)+\operatorname{tr}\left(\left[L, L^{\prime}\right] \cdot\left[\omega, L^{\prime \prime}\right]\right) .
$$

Сначала рассмотрим второе слагаемое. Поскольку след произведения матриц инвариантная форма на алгебре Ли, оно равно

$$
\operatorname{tr}\left(\left[L^{\prime \prime},\left[L, L^{\prime}\right]\right] \cdot \omega\right) .
$$

Циклически переставляя $L, L^{\prime}, L^{\prime \prime}$ и суммируя, получим нуль благодаря тождеству Якоби. Первое слагаемое в (3.9) равно

$$
\operatorname{tr}\left(L \cdot L^{\prime} \cdot d L^{\prime \prime}-L^{\prime} \cdot L \cdot d L^{\prime \prime}\right)
$$

Циклически переставляя $L, L^{\prime}, L^{\prime \prime}$ и суммируя результаты, получаем (используя свойства следа) точную форму

$$
d\left(\operatorname{tr}\left(L \cdot L^{\prime} \cdot L^{\prime \prime}\right)-\operatorname{tr}\left(L \cdot L^{\prime \prime} \cdot L^{\prime}\right)\right)
$$

Ее интеграл по циклу равен нулю, и условие (3.1) проверено. 
ПРЕДЛОЖЕНИЕ 3.2. (а) Кочикл $\gamma_{2, \omega, C}$ не зависит от выбора формы связности $\omega$.

(b) Класс когомологий коцикла $\left[\gamma_{1, \omega, C}\right]$ не зависит от выбора формы связности $\omega$. Точнее,

$$
\gamma_{1, \omega, C}\left(L, L^{\prime}\right)-\gamma_{1, \omega^{\prime}, C}\left(L, L^{\prime}\right)=\frac{1}{2 \pi \mathrm{i}} \int_{C} \operatorname{tr}\left(\left(\omega-\omega^{\prime}\right)\left[L, L^{\prime}\right]\right) .
$$

ДокАЗАТЕЛЬство. Независимость $\gamma_{2, \omega, C}$ от $\omega$ вытекает из доказательства предложения 3.1 .

Пусть $\omega$ и $\omega^{\prime}-$ две формы связности. Положим $\theta=\omega-\omega^{\prime}$. Тогда

$$
\begin{aligned}
\gamma_{1, \omega, C}\left(L, L^{\prime}\right)-\gamma_{1, \omega^{\prime}, C}\left(L, L^{\prime}\right) & =\frac{1}{2 \pi \mathrm{i}} \int_{C} \operatorname{tr}\left(L \cdot\left(\nabla^{(\omega)}-\nabla^{\left(\omega^{\prime}\right)}\right) L^{\prime}\right) \\
& =\frac{1}{2 \pi \mathrm{i}} \int_{C} \operatorname{tr}\left(L \cdot\left[\theta, L^{\prime}\right]\right) d z .
\end{aligned}
$$

Из свойства следа мы получаем

$$
\operatorname{tr}\left(L \cdot\left[\theta, L^{\prime}\right]\right)=\operatorname{tr}\left(L \cdot \theta \cdot L^{\prime}-L \cdot L^{\prime} \cdot \theta\right)=-\operatorname{tr}\left(\theta \cdot\left(L \cdot L^{\prime}-L^{\prime} \cdot L\right)\right)=-\operatorname{tr}\left(\theta \cdot\left[L, L^{\prime}\right]\right) .
$$

Определяя линейную форму

$$
\psi_{\theta, C}(L):=\frac{1}{2 \pi \mathrm{i}} \int_{C} \operatorname{tr}(\theta \cdot L)
$$

на $\overline{\mathfrak{g}}$, видим, что

$$
\gamma_{1, \omega, C}\left(L, L^{\prime}\right)-\gamma_{1, \omega^{\prime}, C}\left(L, L^{\prime}\right)=\psi_{-\theta, C}\left(\left[L, L^{\prime}\right]\right)
$$

Следовательно, разность является кограницей, что и требовалось доказать.

ЗАМЕЧАНИЕ 3.3. Используя (3.7) и (3.15), коцикл $\gamma_{1, \omega, C}$ можно переписать в виде

$$
\gamma_{1, \omega, C}\left(L, L^{\prime}\right)=\frac{1}{2 \pi \mathrm{i}} \int_{C} \operatorname{tr}\left(L d L^{\prime}-\omega \cdot\left[L, L^{\prime}\right]\right) .
$$

В этой форме он впервые определен и изучен в [1] (где $C$ - контур, окружающий $\left.P_{+}\right)$.

Так как коцикл $\gamma_{2, \omega, C}$ не зависит от $\omega$, мы опустим $\omega$ в его обозначении. Заметим, что $\gamma_{2, C}$ обращается в нуль на $\overline{\mathfrak{g}}$ для $\mathfrak{g}=\mathfrak{s l}(n), \mathfrak{s o}(n), \mathfrak{s p}(2 n)$, но не на $\overline{\mathfrak{s}}(n)$, а значит, и не на $\overline{\mathfrak{g l}}(n)$.

3.2. $\mathscr{L}$-инвариантные коциклы. Напомним, что после фиксации формы связности $\omega^{\prime}$ алгебра векторных полей $\mathscr{L}$ действует на $\overline{\mathfrak{g}}$ ковариантными производными $\nabla_{e}^{\left(\omega^{\prime}\right)}$. Позднее мы будем предполагать, что $\omega=\omega^{\prime}$.

ОПРЕДЕЛЕниЕ 3.4 . Коцикл на $\overline{\mathfrak{g}}$ называется $\mathscr{L}$-инвариантным (по отношению к $\left.\omega^{\prime}\right)$, если

$$
\gamma\left(\nabla_{e}^{\left(\omega^{\prime}\right)} L, L^{\prime}\right)+\gamma\left(L, \nabla_{e}^{\left(\omega^{\prime}\right)} L^{\prime}\right)=0 \quad \forall e \in \mathscr{L}, \quad \forall L, L^{\prime} \in \overline{\mathfrak{g}} .
$$




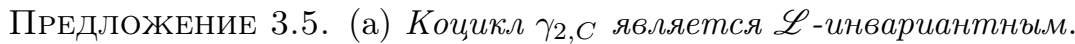

(b) Если $\omega=\omega^{\prime}$, то коцикл $\gamma_{1, \omega, C}$ также $\mathscr{L}$-инвариантен.

ДокАЗАТЕЛЬСтво. Поскольку коциклы антисимметричны, $\mathscr{L}$-инвариантность записывается как

$$
\gamma\left(\nabla_{e}^{\left(\omega^{\prime}\right)} L, L^{\prime}\right)=\gamma\left(\nabla_{e}^{\left(\omega^{\prime}\right)} L^{\prime}, L\right) \quad \forall e \in \mathscr{L}, \quad \forall L, L^{\prime} \in \overline{\mathfrak{g}} .
$$

Ниже мы используем локальную запись $e=\tilde{e} \frac{d}{d z}, \omega=\widetilde{\omega} d z$ и $\omega^{\prime}=\widetilde{\omega}^{\prime} d z$.

Сначала мы рассмотрим $\gamma_{2, C}$. Для подынтегральной формы в левой части (3.20) имеем

$$
\operatorname{tr}\left(\nabla_{e}^{\left(\omega^{\prime}\right)} L\right) \cdot \operatorname{tr}\left(\nabla^{(\omega)} L^{\prime}\right)=\operatorname{tr}(e . L) \cdot \operatorname{tr}\left(\frac{d L^{\prime}}{d z} d z\right)=\tilde{e} \cdot d z \cdot \operatorname{tr}\left(\frac{d L}{d z}\right) \cdot \operatorname{tr}\left(\frac{d L^{\prime}}{d z}\right) .
$$

Перестановка $L$ и $L^{\prime}$ не меняет выражения. Следовательно, коцикл $\gamma_{2, C}$ является $\mathscr{L}$-инвариантным.

Далее рассмотрим $\gamma_{1, \omega, C}$. Для подынтегральной формы имеем

$$
\begin{aligned}
\operatorname{tr}\left(\nabla_{e}^{\left(\omega^{\prime}\right)} L \cdot \nabla^{(\omega)} L^{\prime}\right) & =\operatorname{tr}\left(\left(\frac{d L}{d z} \tilde{e}+\left[\widetilde{\omega}^{\prime} \cdot e, L\right]\right)\left(\frac{d L^{\prime}}{d z}+\left[\widetilde{\omega}, L^{\prime}\right]\right)\right) \\
& =\tilde{e} \cdot d z \cdot \operatorname{tr}\left(\left(\frac{d L}{d z}+\left[\widetilde{\omega}^{\prime}, L\right]\right)\left(\frac{d L^{\prime}}{d z}+\left[\widetilde{\omega}, L^{\prime}\right]\right)\right) .
\end{aligned}
$$

Так как $\omega=\omega^{\prime}$, это выражение также инвариантно относительно перестановки $L$ и $L^{\prime}$, что и требовалось доказать.

В случае, когда $\mathfrak{g}$ проста и цикл $C$, по которому ведется интегрирование, разделяющий (см. п. 3.4), в утверждении (b) мы даже имеем “и только если", см. предложение 3.13 .

Назовем класс когомологий $\mathscr{L}$-инвариантным, если в нем есть $\mathscr{L}$-инвариантный коцикл. Следует предупредить читателя, что это не означает, что все представители этого класса являются $\mathscr{L}$-инвариантными коциклами. Напротив, в теореме 3.9 мы покажем, что, с точностью до скалярного множителя, существует самое большее один $\mathscr{L}$-инвариантный представляющий коцикл. Очевидно, что $\mathscr{L}$-инвариантные классы образуют подпространство в $\mathrm{H}^{2}(\overline{\mathfrak{g}}, \mathbb{C})$, которое мы обозначим $\mathrm{H}_{\mathscr{L}}^{2}(\overline{\mathfrak{g}}, \mathbb{C})$.

3.3. Замечания о коциклах на $\mathscr{D} \mathfrak{g}$. Ниже положим $\omega=\omega^{\prime}$. Свойство $\mathscr{L}$-инвариантности дает весьма элегантный способ выделения единственного элемента в классе когомологий. Но оно имеет более общее значение. В п. 2.4 мы ввели алгебру $\mathscr{D}_{\mathfrak{g}}^{1}$. Алгебра операторов Лакса является подалгеброй этой последней. Если дан 2-коцикл $\gamma$ на $\overline{\mathfrak{g}}$, мы можем продолжить его как билинейную форму на $\mathscr{D} \mathfrak{g}$, полагая для $L, L^{\prime} \in \overline{\mathfrak{g}}, e, f \in \mathscr{L}$

$$
\widetilde{\gamma}\left(L, L^{\prime}\right)=\gamma\left(L, L^{\prime}\right), \quad \widetilde{\gamma}(e, L)=\widetilde{\gamma}(L, e)=0, \quad \widetilde{\gamma}(e, f)=0 .
$$

ПредложениЕ 3.6. Билинейная форма $\widetilde{\gamma}$ является коииклом на $\mathscr{D}_{\mathfrak{g}}^{1}$ тогда и только тогда, когда кочикл $\gamma$ является $\mathscr{L}$-инвариантным. 
ДокАзАТЕЛЬство. Очевидно, что соотношение, определяющее коцикл, выполняется для троек элементов, состоящих только из токов или только из векторных полей. Единственное условие в определении коцикла, которое для $\widetilde{\gamma}$ не вытекает автоматически из (3.23) - это

$$
\widetilde{\gamma}\left(\left[L, L^{\prime}\right], e\right)+\widetilde{\gamma}\left(\left[L^{\prime}, e\right], L\right)+\widetilde{\gamma}\left([e, L], L^{\prime}\right)=0 .
$$

Воспользовавшись (2.56), получим, что (3.24) выполняется тогда и только тогда, когда

$$
\gamma\left(\nabla_{e}^{(\omega)} L, L^{\prime}\right)+\gamma\left(L, \nabla_{e}^{(\omega)} L^{\prime}\right)=0 .
$$

Отсюда вытекает требуемое утверждение.

В [20] было показано, что для алгебр токов Кричевера-Новикова обратное также верно в следующем смысле: каждый локальный коцикл (определение см. в следующем пункте) на $\mathscr{D}_{\mathfrak{g}}^{1}$ когомологичен локальному кочиклу, ограничение которого на $\overline{\mathfrak{g}}$ является $\mathscr{L}$-инвариантным. Таким образом, коциклы, полученные из проективных представлений $\overline{\mathfrak{g}}$, допускающих продолжение до проективных представлений алгебры $\mathscr{D}_{\mathfrak{g}}^{1}$, дают $\mathscr{L}$-инвариантные коциклы с точностью до кограниц.

Аналогичные утверждения верны для алгебры $\mathscr{D}_{\mathfrak{g}}^{1}$, ассоциированной с алгеброй операторов Лакса $\overline{\mathfrak{g}}$. Детальное изложение будет опубликовано в другом месте.

3.4. Локальные коциклы. Коцикл $\gamma$ на почти градуированной алгебре Ли $\mathscr{V}$ называется локальным, если существуют $R, S \in \mathbb{Z}$ такие, что (см. [7])

$$
\gamma\left(\mathscr{V}_{n}, \mathscr{V}_{m}\right) \neq 0 \Rightarrow R \leqslant n+m \leqslant S
$$

Важность этого понятия обусловлена тем, что именно для локальных коциклов почти градуировка может быть продолжена с $\mathscr{V}$ на соответствующее центральное расширение $\widehat{\mathscr{V}}_{\gamma}$ путем присвоения какой-либо степени (обычно нулевой) центральному элементу.

Мы называем класс когомологий локалъным, если он содержит локальный коцикл. Повторимся, что не каждый коцикл в локальном классе локален. Очевидно, что множество локальных классов когомологий является подпространством пространства $\mathrm{H}^{2}(\overline{\mathfrak{g}}, \mathbb{C})$, и мы обозначим его $\mathrm{H}_{\mathrm{loc}}^{2}(\overline{\mathfrak{g}}, \mathbb{C})$. Это пространство классифицирует почти градуированные центральные расширения алгебры $\overline{\mathfrak{g}}$ с точностью до эквивалентности. Классы когомологий, допускающие локальный и $\mathscr{L}$-инвариантный представляющий коцикл, образуют подпространство пространства $\mathrm{H}_{\mathrm{loc}}^{2}(\overline{\mathfrak{g}}, \mathbb{C})$, и мы обозначаем его $\mathrm{H}_{\mathrm{loc}, \mathscr{L}}^{2}(\overline{\mathfrak{g}}, \mathbb{C})$.

Для общего контура интегрирования $C$ коциклы $\gamma_{2, C}$ и $\gamma_{1, \omega, C}$ не являются ни локальными, ни определяющими локальный класс когомологий. Но если мы выберем для интегрирования контур $C_{s}$, отделяющий $P_{+}$от $P_{-}$, то, как мы сейчас покажем, они являются локальными (для $\gamma_{1, \omega, C}$ это впервые сделано в [1]). Такие контуры гомологичны малому контуру, окружающему $P_{+}$. В случае, когда мы интегрируем по этому последнему, мы опускаем указание на него в обозначении $\gamma$. В этом случае интеграл может быть заменен соответствующим вычетом в точке $P_{+}$. 
ПРЕДЛОЖЕНИЕ 3.7. 1-форма $\operatorname{tr}\left(L \cdot \nabla^{(\omega)} L^{\prime}\right)$ не имеет полюсов, кроме, возможно, полюсов в $P_{ \pm}$. Коцикл

$$
\gamma_{1, \omega}\left(L, L^{\prime}\right)=\operatorname{res}_{P_{+}}\left(\operatorname{tr}\left(L \cdot \nabla^{(\omega)} L^{\prime}\right)\right)
$$

локален. Он также $\mathscr{L}$-инвариантен при условии, что $\omega$ совпадает с формой связности $\omega^{\prime}$, определяющей действие $\mathscr{L}$.

ДоказАтельство. Как отмечено выше, коцикл $\gamma_{1, \omega}$ может быть записан в виде (3.18). В этой форме его локальность установлена в [1] (теоремы 4.3, 4.6 и 4.9). $\mathscr{L}$-инвариантность следует из предложения 3.5 .

ПРЕДЛОЖЕНИЕ 3.8. 1-борма $\operatorname{tr}(L) \cdot \operatorname{tr}\left(d L^{\prime}\right)$ не имеет полюсов, кроме, возможно, полюсов в $P_{ \pm}$. Коцикл

$$
\gamma_{2}\left(L, L^{\prime}\right)=\operatorname{res}_{P_{+}}\left(\operatorname{tr}(L) \cdot \operatorname{tr}\left(d L^{\prime}\right)\right)
$$

локален и $\mathscr{L}$-инвариантен.

ДоКАЗАТЕЛЬСтво. Матрицы из $\overline{\mathfrak{s l}}(n), \overline{\mathfrak{s o}}(n)$ и $\overline{\mathfrak{s p}}(2 n)$ имеют нулевой след, следовательно, для них коцикл обращается в нуль. Остается рассмотреть $\overline{\mathfrak{g l}}(n)$. Положим $h=\operatorname{tr}(L) \cdot \operatorname{tr}\left(d L^{\prime}\right)$; это мероморфный дифференциал.

Согласно (2.4), мы имеем в точках $P_{s} \in W$

$$
L=\frac{L_{s,-1}}{z_{s}}+\sum_{k \geqslant 0} L_{s, k} z^{k}, \quad d L^{\prime}=\left(\frac{-L_{s,-1}^{\prime}}{z_{s}^{2}}+\sum_{k \geqslant 1} L_{s, k}^{\prime} k z^{k-1}\right) d z .
$$

По условию $(2.5) \operatorname{tr}\left(L_{s,-1}\right)=\operatorname{tr}\left(L_{s,-1}^{\prime}\right)=0$. Следовательно, ни $\operatorname{tr}(L)$, ни $\operatorname{tr}\left(d L^{\prime}\right)$ не имеют полюсов в точках множества $W$, а значит, $h$ не имеет полюсов, кроме, возможно, полюсов в точках $P_{ \pm}$. Отсюда, в частности, вытекает, что вычет $h$ в $P_{+}$равен вычету в $P_{-}$с обратным знаком.

Пусть $L$ и $L^{\prime}$ однородны. Порядок $h$ в $P_{+}$ограничен снизу числом $\operatorname{ord}_{P_{+}}(L)+$ $\operatorname{ord}_{P_{+}}\left(L^{\prime}\right)-1$. Следовательно, $h$ не имеет вычета в $P_{+}$при $\operatorname{deg} L+\operatorname{deg} L^{\prime}>0$.

Используя (2.14) и рассматривая порядки в точке $P_{-}$, мы видим, что существует такая константа $S$, что если $\operatorname{deg} L+\operatorname{deg} L^{\prime}<S$, то $h$ не имеет полюса в $P_{-}$. Это доказывает локальность. $\mathscr{L}$-инвариантность установлена предложением 3.5 .

\section{5. Основная теорема.}

Tеорема 3.9. (а) Ecлu $\mathfrak{g}$ nроста (m. e. $\overline{\mathfrak{g}}=\mathfrak{s l}(n), \mathfrak{s o}(n), \mathfrak{s p}(2 n))$, mo пространство локальных классов когомологий одномерно. Если мы выберем форму связности $\omega$, то это пространство будет порождено классом когомологий кочикла $\gamma_{1, \omega}$. Каждый $\mathscr{L}$-инвариантный (по отношению к связности $\omega$ ) локальный коцикл кратен кочиклу $\gamma_{1, \omega}$.

(b) Для $\overline{\mathfrak{g}}=\overline{\mathfrak{g l}}$ пространство локальных классов когомологий, ограничение которых на скалярную подалгебру $\mathscr{L}$-инвариантно, двумерно. Если мъ въберем форму связности $\omega$, то это пространство будет порождено классами когомологий коциклов $\gamma_{1, \omega}$ и $\gamma_{2}$. Каждый $\mathscr{L}$-инвариантный локальный коцикл является линейной комбинацией кочиклов $\gamma_{1, \omega} u \gamma_{2}$. 
ЗАмЕчАниЕ 3.10. Любые два когомологичных коцикла алгебры $\overline{\mathfrak{g l}}$ имеют одно и то же ограничение на скалярную подалгебру, поскольку последняя коммутативна. Требование пункта (b) теоремы 3.9 означает, что это ограничение $\mathscr{L}$-инвариантно.

ДоКАЗАТЕЛЬСТВо тЕОРЕмы. Здесь мы дадим общий план доказательства. Техническая часть откладывается до разделов 4,5 .

Из предложений 4.8 и 4.10 вытекает, что $\mathscr{L}$-инвариантные и локальные коциклы обязательно являются линейными комбинациями указанного вида. Это доказывает теорему для пространства когомологий $\mathrm{H}_{\mathrm{loc}, \mathscr{L}}(\overline{\mathfrak{g}}, \mathbb{C})$. Для скалярной подалгебры это все, что требуется доказать, так как мы включили $\mathscr{L}$-инвариантность в условие теоремы. Для полупростых алгебр нам предстоит показать, что в каждом локальном классе когомологий имеется $\mathscr{L}$-инвариантный представитель. Но по теореме 5.1 пространство $\mathrm{H}_{\mathrm{loc}}(\overline{\mathfrak{g}}, \mathbb{C})$ самое большее одномерно. Поскольку ввиду предложения 3.12 локальный коцикл $\gamma_{1, \omega}$ не является кограницей, это пространство одномерно и $\gamma_{1, \omega}-$ его нетривиальный элемент.

СлЕДСТвИЕ 3.11. Пусть $\mathfrak{g}$ - простая классическая алгебра Ли и $\overline{\mathfrak{g}}-$ ас-

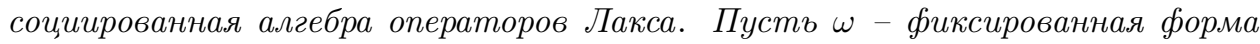
связности. Тогда в каждом классе $[\gamma] \in \mathrm{H}_{\mathrm{loc}}(\overline{\mathfrak{g}}, \mathbb{C})$ существует единственный представитель, который локален и $\mathscr{L}$-инвариантен (относительно $\omega)$. Более того, $\gamma^{\prime}=\alpha \gamma_{1, \omega}$, где $\alpha \in \mathbb{C}$.

ПРЕДЛОЖЕНИЕ 3.12. Коцикл $\gamma=\gamma_{1, \omega}$ не является кограницей.

ДокАЗАТЕЛЬСтво. Предположим, что $\gamma-$ кограница. Это означало бы, что существует линейная форма $\phi: \overline{\mathfrak{g}} \rightarrow \mathbb{C}$ такая, что

$$
\gamma\left(L, L^{\prime}\right)=\operatorname{res}_{P_{+}} \operatorname{tr}\left(L \cdot \nabla L^{\prime}\right)=\phi\left(\left[L, L^{\prime}\right]\right) .
$$

Возьмем $H \in \mathfrak{h}$ так, что $\kappa(H, H) \neq 0$, где $\mathfrak{h}$ - картановская подалгебра простой части алгебры $\mathfrak{g}, \kappa$ - форма Картана-Киллинга. Пусть $H_{0} \in \overline{\mathfrak{g}}-$ элемент, определенный условием (2.16). В частности, $H_{0}=H+O\left(z_{+}\right)$. Положим ${ }^{1}$ $H_{(n)}=H_{0} \cdot A_{n} \in \overline{\mathfrak{g}}$, следовательно, $H_{(n)}=H \cdot A_{n}+O\left(z_{+}^{n+1}\right)$. Пусть ниже $n \neq 0$. Мы имеем

$$
\nabla H_{(n)}=\nabla\left(H_{0} \cdot A_{n}\right)=\nabla\left(H_{0}\right) \cdot A_{n}+H_{0} d A_{n} .
$$

Выражение $\nabla H_{0}$ имеет неотрицательный порядок, $A_{n}$ - порядок $n, H_{0}$ - порядок 0 и $d A_{n}-$ порядок $n-1$ в точке $P_{+}$. Следовательно,

$$
\nabla H_{(n)}=H_{0} d A_{n}+O\left(z_{+}^{n}\right) d z_{+} .
$$

Далее,

$$
\begin{aligned}
\gamma\left(H_{(-1)}, H_{(1)}\right) & =\operatorname{res}_{P_{+}} \operatorname{tr}\left(H_{(-1)} \cdot \nabla H_{(1)}\right)=\operatorname{res}_{P_{+}} \operatorname{tr}\left(H_{0} A_{-1} H_{0} d A_{1}\right) \\
& =\operatorname{res}_{P_{+}} \operatorname{tr}\left(H_{0}^{2} \frac{d z_{+}}{z_{+}}\right) .
\end{aligned}
$$

\footnotetext{
1Заметим, что $H_{(n)}$ и $H_{n}$, вообе говоря, различны, но совпадают с точностью до членов достаточно высокого порядка.
} 
Так как $H_{0}^{2}=H^{2}+O\left(z_{+}\right)$, мы получаем

$$
\gamma\left(H_{(-1)}, H_{(1)}\right)=\operatorname{res}_{P_{+}}\left(\operatorname{tr}\left(H^{2}\right) \frac{d z_{+}}{z_{+}}\right)=\operatorname{tr}\left(H^{2}\right)=\alpha \kappa(H, H) \neq 0,
$$

где $\alpha$ - ненулевой коэффициент пропорциональности между формой, определенной следом, и формой Картана-Киллинга. Но

$$
\left[H_{(-1)}, H_{(1)}\right]=\left[H_{0} A_{-1}, H_{0} A_{1}\right]=\left[H_{0}, H_{0}\right] A_{-1} A_{1}=0 .
$$

Соотношения (3.34) и (3.35) противоречат предположению (3.30). Предложение 3.12 доказано.

ПРЕДЛОжЕНИЕ 3.13. (а) Пусть $\gamma$-локальный и $\mathscr{L}$-инвариантный кочикл, который является кограницей, тогда $\gamma=0$.

(b) Пусть $\mathfrak{g}$ - простая, тогда коиикл $\gamma_{1, \omega^{\prime}}$ является $\mathscr{L}$-инвариантным по отношению $\kappa$ форме связности $\omega$ тогда и только тогда, когда $\omega=\omega^{\prime}$.

ДоказАтельство. (а) По теореме 3.9 мы получаем $\gamma=\alpha \gamma_{1, \omega}+\beta \gamma_{2}$, где $\beta=0$ в случае, когда $\mathfrak{g}$ проста. Но ни один из этих коциклов не является кограницей. Следовательно, $\alpha=\beta=0$.

(b) Поскольку $\gamma_{1, \omega}$ и $\gamma_{1, \omega^{\prime}}$ локальны и $\mathscr{L}$-инвариантны по отношению к $\omega$, их разность $\gamma_{1, \omega}-\gamma_{1, \omega^{\prime}}$ тоже обладает этими свойствами. Ввиду предложения 3.2 она является границей. Следовательно, согласно утверждению (а) настоящего предложения, $\gamma_{1, \omega}-\gamma_{1, \omega^{\prime}}=0$. Воспользуемся явным выражением (3.13) для этой разности. Предположим, что $\omega \neq \omega^{\prime}$. Пусть $m$ - порядок элемента

$$
\theta=\omega-\omega^{\prime}=\left(\theta_{m} z_{+}^{m}+O\left(z_{+}^{m}\right)\right) d z_{+}
$$

в точке $P_{+}$. Так как $\mathfrak{g}$ проста, то билинейная форма $\operatorname{tr}(A \cdot B)$ невырождена, и мы находим

$$
\widehat{\theta}=\widehat{\theta}_{-m-1} z^{-m-1}+O\left(z_{+}^{-m}\right),
$$

так что $\beta=\operatorname{tr}\left(\theta_{m} \cdot \widehat{\theta}_{-m-1}\right) \neq 0$. По лемме 2.6 имеем $\widehat{\theta}=\left[L, L^{\prime}\right]+L^{\prime \prime}$, где $\operatorname{ord}\left(L^{\prime \prime}\right) \geqslant-m$. Следовательно,

$$
\begin{aligned}
0 \neq \beta & =\operatorname{tr}\left(\theta_{m} \cdot \widehat{\theta}_{-m-1}\right)=\frac{1}{2 \pi \mathrm{i}} \int_{C_{s}} \operatorname{tr}\left(\left(\omega-\omega^{\prime}\right) \cdot\left(\left[L, L^{\prime}\right]+L^{\prime \prime}\right)\right) \\
& =\frac{1}{2 \pi \mathrm{i}} \int_{C_{s}} \operatorname{tr}\left(\left(\omega-\omega^{\prime}\right) \cdot\left[L, L^{\prime}\right]\right)=\gamma_{1, \omega}\left(L, L^{\prime}\right)-\gamma_{1, \omega^{\prime}}\left(L, L^{\prime}\right)=0,
\end{aligned}
$$

что является противоречием.

\section{4. Единственность $\mathscr{L}$-инвариантных коциклов}

4.1. Общая индукция. Напомним, что $\overline{\mathfrak{g}}$ разлагается на подпространства однородных элементов степени $n: \overline{\mathfrak{g}}=\bigoplus_{n \in \mathbb{Z}} \overline{\mathfrak{g}}_{n}$. Подпространство $\overline{\mathfrak{g}}_{n}$ имеет базис $\left\{L_{n}^{r} \mid r=1, \ldots, \operatorname{dim} \mathfrak{g}\right\}$.

Пусть ниже $\gamma-\mathscr{L}$-инвариантный коцикл на $\overline{\mathfrak{g}}$. Мы предполагаем только, что он ограничен сверху, т. е. существует $K$ (не зависящее от $n$ и $m$ ) такое, что 
из $\gamma\left(\overline{\mathfrak{g}}_{n}, \overline{\mathfrak{g}}_{m}\right) \neq 0$ следует $n+m \leqslant K$. Далее, напомним, что наша связность $\omega$, необходимая для определения действия $\mathscr{L}$ на $\overline{\mathfrak{g}}$, выбрана голоморфной в точке $P_{+}$.

Для пары $\left(L_{n}^{r}, L_{m}^{s}\right)$ однородных элементов назовем число $n+m$ ее уровнем. Следуя стратегии, разработанной в [21], мы рассмотрим значения коцикла $\gamma\left(L_{n}^{r}, L_{m}^{s}\right)$ на парах уровня $l=n+m$ и проведем индукцию по уровню. Ввиду ограниченности сверху, значения коцикла обратятся в нуль на всех парах достаточно большого уровня, и окажется, что все определяется значениями коцикла на нулевом уровне. В заключение мы докажем единственность коцикла с точностью до нормировки на нулевом уровне.

Для значений коцикла $\gamma$ на парах элементов уровня $l$ и их линейных комбинаций символ $\equiv$ обозначает равенство с точностью до значений коцикла $\gamma$ на более высоких уровнях. Строго это надо понимать в следующем смысле:

$$
\sum \alpha_{r, s}^{n} \gamma\left(L_{n}^{r}, L_{l-n}^{s}\right) \equiv 0, \quad \alpha_{r, s}^{n} \in \mathbb{C},
$$

означает сравнение по модулю линейной комбинации значений $\gamma$ на парах базисных элементов уровней $l^{\prime}>l$. Коэффициенты этой линейной комбинации, как и $\alpha_{r, s}^{n}$, зависят только от структуры алгебры $\overline{\mathfrak{g}}$ и не зависят от $\gamma$.

Мы будем также использовать символ $\equiv$ для равенств в алгебре $\overline{\mathfrak{g}}$, справедливых по модулю членов более высокого порядка по сравнению с выписанными.

Ввиду $\mathscr{L}$-инвариантности имеем

$$
\gamma\left(\nabla_{e_{p}} L_{m}^{r}, L_{n}^{s}\right)+\gamma\left(L_{m}^{r}, \nabla_{e_{p}} L_{n}^{s}\right)=0
$$

Используя почти градуированную структуру (2.48), получим следующую полезную формулу:

$$
m \gamma\left(L_{p+m}^{r}, L_{n}^{s}\right)+n \gamma\left(L_{m}^{r}, L_{n+p}^{s}\right) \equiv 0,
$$

справедливую для всех $n, m, p \in \mathbb{Z}$.

ПрЕДЛОЖЕНИЕ 4.1. Пусть $m+n \neq 0$, тогда на уровне $m+n$ имеем

$$
\gamma\left(\overline{\mathfrak{g}}_{m}, \overline{\mathfrak{g}}_{n}\right) \equiv 0
$$

ДокаЗАТЕЛЬСтво. Из (4.3) при $p=0$ вытекает, что

$$
(m+n) \gamma\left(L_{m}^{r}, L_{n}^{s}\right) \equiv 0 .
$$

Следовательно, при $m+n \neq 0$ приходим к нужному результату: $\gamma\left(L_{m}^{r}, L_{n}^{s}\right) \equiv 0$.

ПРЕДЛОЖЕНИЕ 4.2. Для любого $m \in \mathbb{Z}$

$$
\gamma\left(L_{m}^{r}, L_{0}^{s}\right) \equiv 0
$$

ДокАЗАТЕЛЬСтво. Вычисляя (4.3) для $m=1$ и $n=0$, получаем требуемое.

ПреДЛОЖЕНИЕ 4.3. (а) Если $n+m>0$, mo $\gamma\left(\overline{\mathfrak{g}}_{n}, \overline{\mathfrak{g}}_{m}\right)=0$, m.е. коцикл ограничен сверху нулем.

(b) Eсли $\gamma\left(\overline{\mathfrak{g}}_{n}, \overline{\mathfrak{g}}_{-n}\right)=0$, то коцикл $\gamma$ тождественно равен нулю. 
ДокАзАТЕЛЬство. Если $\gamma=0$, то утверждение тривиально. Предположим, что $\gamma \neq 0$. Так как $\gamma$ ограничен сверху, то существует наименьшая верхняя граница $l$ такая, что на уровнях выше $l$ коцикл равен нулю. Предположим, что $l>0$, тогда в условиях предложения 4.1 значения на уровне $l$ являются выражениями от значений на уровнях выше $l$. На последних коцикл равен нулю, значит, он равен нулю и на уровне $l$. Это противоречие доказывает утверждение (а).

По индукции с использованием предложения 4.1 получим, что если коцикл равен нулю на уровне 0, то он нулевой. Это доказывает (b).

Комбинируя предложения 4.2 и 4.3, мы получим

СлЕДСТВиЕ 4.4. Для любого $m \geqslant 0$

$$
\gamma\left(L_{m}^{r}, L_{0}^{s}\right)=0
$$

ПРЕДЛОЖЕНИЕ 4.5. Справедливы равенства

$$
\begin{aligned}
& \gamma\left(L_{n}^{r}, L_{-n}^{s}\right)=n \gamma\left(L_{1}^{r}, L_{-1}^{s}\right), \\
& \gamma\left(L_{1}^{r}, L_{-1}^{s}\right)=\gamma\left(L_{1}^{s}, L_{-1}^{r}\right) .
\end{aligned}
$$

ДокаЗАтеЛьСтво. Возьмем в (4.3) $n=-k, m=1$ и $p=k-1$. Это даст выражение (4.8) с точностью до членов более высокого уровня. Но так как мы на нулевом уровне, члены более высокого уровня обращаются в нуль. Полагая $n=-1$, получим (4.9).

Суммируем результаты, полученные к настоящему моменту. Независимо от структуры алгебры Ли $\mathfrak{g}$ мы получили для произвольного $\mathscr{L}$-инвариантного и ограниченного коцикла $\gamma$ следующее:

1) коцикл ограничен сверху нулем;

2) коцикл однозначно задается своими значениями на нулевом уровне;

3) на нулевом уровне коцикл однозначно задается значениями $\gamma\left(L_{1}^{r}, L_{-1}^{s}\right)$ при $r, s=1, \ldots, \operatorname{dim} \mathfrak{g} ;$

4) остальные значения коцикла на нулевом уровне задаются соотношениями (4.8) и $\gamma\left(L_{0}^{r}, L_{0}^{s}\right)=0$.

Пусть $X \in \mathfrak{g}$, обозначим через $\widetilde{X}_{n}$ любой элемент в $\overline{\mathfrak{g}}$ с главным членом $X z_{+}^{n}$ разложения в $P_{+}$. Определим отображение

$$
\psi_{\gamma}: \mathfrak{g} \times \mathfrak{g} \rightarrow \mathbb{C}, \quad \psi_{\gamma}(X, Y)=\gamma\left(\widetilde{X}_{1}, \tilde{Y}_{-1}\right) .
$$

Поскольку коцикл обращается в нуль на уровнях выше нуля, $\psi_{\gamma}$ не зависит от выбора $\widetilde{X}_{1}$ и $\widetilde{Y}_{-1}$. Очевидно, что $\psi_{\gamma}$ является билинейной формой на $\mathfrak{g}$.

ПреДлОЖение 4.6. (а) $\psi_{\gamma}$ симметрична, m.е. $\psi_{\gamma}(X, Y)=\psi_{\gamma}(Y, X)$.

(b) $\psi_{\gamma}$ инвариантна, m.е.

$$
\psi_{\gamma}([X, Y], Z)=\psi_{\gamma}(X,[Y, Z]) .
$$

ДокАЗАТЕЛьСтво. Ввиду (4.9) мы имеем

$$
\psi_{\gamma}(X, Y)=\gamma\left(\widetilde{X}_{1}, \widetilde{Y}_{-1}\right)=\gamma\left(\widetilde{Y}_{1}, \widetilde{X}_{-1}\right)=\psi_{\gamma}(Y, X) .
$$

Это доказывает симметричность. 
Далее, пользуясь свойством $\left.\left[\widetilde{X}_{1}, \widetilde{Y}_{0}\right] \equiv \widetilde{[X, Y}\right]_{1}$, тем, что коцикл обращается в нуль на положительных уровнях, и определением коцикла, имеем

$$
\begin{aligned}
\psi_{\gamma}([X, Y], Z) & =\gamma\left(\widetilde{[X, Y}_{1}, \widetilde{Z}_{-1}\right)=\gamma\left(\left[\widetilde{X}_{1}, \widetilde{Y}_{0}\right], \widetilde{Z}_{-1}\right) \\
& =-\gamma\left(\left[\widetilde{Y}_{0}, \widetilde{Z}_{-1}\right], \widetilde{X}_{1}\right)-\gamma\left(\left[\widetilde{Z}_{-1}, \widetilde{X}_{1}\right], \widetilde{Y}_{0}\right)
\end{aligned}
$$

Последний член обращается в нуль согласно следствию 4.4. Следовательно,

$$
\psi_{\gamma}([X, Y], Z)=\gamma\left(\tilde{X}_{1},\left[\tilde{Y}_{0}, \widetilde{Z}_{-1}\right]\right)=\gamma\left(\tilde{X}_{1}, \widetilde{[Y, Z]}-1\right)=\psi_{\gamma}(X,[Y, Z]) .
$$

Предложение 4.6 доказано.

Поскольку коцикл $\gamma$ фиксирован значениями $\gamma\left(L_{1}^{r}, L_{-1}^{s}\right)$, а последние фиксированы билинейным отображением $\psi_{\gamma}$, мы доказали следующее утверждение.

Теорема 4.7. Пусть $\gamma$ является $\mathscr{L}$-инвариантным коииклом на $\overline{\mathfrak{g}}$, ограниченным сверху нулем. Тогда $\gamma$ полностью задан ассоциированной симметричной и инвариантной билинейной формой $\psi_{\gamma}$ на $\mathfrak{g}$, определенной соотношением (4.10).

4.2. Случай простой алгебры $\mathfrak{g}$. По теореме 4.7 коцикл $\gamma$ полностью задан ассоциированной симметричной инвариантной билинейной формой $\psi_{\gamma}$. Для конечномерной простой алгебры Ли каждая такая форма кратна форме Картана-Киллинга $\kappa$. Этим завершается доказательство единственности коцикла. Существование очевидно, так как $\gamma_{1, \omega}$, см. $(3.27)$, является $\mathscr{L}$-инвариантным и локальным коциклом. Следовательно, мы получили, что каждый локальный и $\mathscr{L}$-инвариантный коцикл кратен $\gamma_{1, \omega}$. Ввиду предложения 3.12 коцикл $\gamma_{1, \omega}$ не является кограницей. Мы получаем следующий результат.

ПРЕДЛОЖЕНИЕ 4.8. Пустъ $\mathfrak{g}$ проста, тогда

$$
\operatorname{dim} \mathrm{H}_{\mathrm{loc}, \mathscr{L}}(\overline{\mathfrak{g}}, \mathbb{C})=1,
$$

и это пространство когомологий порождено классом когомологий кочикла $\gamma_{1, \omega}$. Более того, каждый $\mathscr{L}$-инвариантный кочикл, ограниченный сверху, локален.

4.3. Случай $\overline{\mathfrak{g l}}(n)$. Мы имеем разложение в прямую сумму алгебр Ли: $\overline{\mathfrak{g l}}=$ $\overline{\mathfrak{s}}(n) \oplus \overline{\mathfrak{s l}}(n)$. Пусть $\gamma-$ коцикл на $\overline{\mathfrak{g l}}(n)$, обозначим через $\gamma^{\prime}$ и $\gamma^{\prime \prime}$ его ограничения на $\overline{\mathfrak{s}}(n)$ и $\overline{\mathfrak{s l}}(n)$ соответственно.

ПРЕДЛОЖЕНИЕ 4.9. Для любы $x \in \overline{\mathfrak{s}}(n) u y \in \overline{\mathfrak{s l}}(n)$

$$
\gamma(x, y)=0
$$

ДокАзАтельство. Пусть $M$ - верхняя граница для коцикла $\gamma$. Возьмем $x$ и $y$ как выше. В частности, существует $m$ такое, что $x$ можно записать в виде линейной комбинации базисных элементов степени $\geqslant m$. По лемме 2.6 существуют элементы $y_{1}^{(i)}, y_{2}^{(i)} \in \overline{\mathfrak{s l}}(n), \quad i=1, \ldots, k$, и $B \in \overline{\mathfrak{s l}}(n)$, где $B$ - 
линейная комбинация элементов степени не менее $M-m+1$, такие, что $y=$ $\sum_{i=1}^{k}\left[y_{1}^{(i)}, y_{2}^{(i)}\right]+B$. Тогда

$$
\gamma(x, y)=\gamma\left(x, \sum_{i=1}^{k}\left[y_{1}^{(i)}, y_{2}^{(i)}\right]+B\right)=\sum_{i=1}^{k} \gamma\left(x,\left[y_{1}^{(i)}, y_{2}^{(i)}\right]\right)+\gamma(x, B)
$$

Последнее слагаемое обращается в нуль, так как коцикл ограничен числом $M$. Для остального мы получаем, пользуясь определением коцикла,

$$
\gamma\left(x,\left[y_{1}^{(i)}, y_{2}^{(i)}\right]\right)=\gamma\left(\left[y_{2}^{(i)}, x\right], y_{1}^{(i)}\right)+\gamma\left(\left[x, y_{1}^{(i)}\right], y_{2}^{(i)}\right)
$$

Коммутаторы в правой части обращаются в нуль, так как $\overline{\mathfrak{s}}(n)$ и $\overline{\mathfrak{s l}}(n)$ коммутируют. Это доказывает утверждение.

Из этого предложения следует, что $\gamma\left(x_{1}+y_{1}, x_{2}+y_{2}\right)=\gamma\left(x_{1}, x_{2}\right)+\gamma\left(y_{1}, y_{2}\right)$, если $x_{1}, x_{2} \in \overline{\mathfrak{s}}(n)$ и $y_{1}, y_{2} \in \overline{\mathfrak{s l}}(n)$. Следовательно, $\gamma=\gamma^{\prime} \oplus \gamma^{\prime \prime}$. Если $\gamma$ локален и (или) $\mathscr{L}$-инвариантен, то же самое верно для $\gamma^{\prime}$ и $\gamma^{\prime \prime}$.

Сначала мы рассмотрим алгебру $\overline{\mathfrak{s}}(n)$. Она изоморфна $\mathscr{A}$, изоморфизм устанавливается отображением

$$
L \mapsto \frac{1}{n} \operatorname{tr}(L) .
$$

В [21; теорема 4.3] показано, что с точностью до нормировки единственный $\mathscr{L}$-инвариантный коцикл на $\mathscr{A}$ дается выражением

$$
\gamma_{\mathscr{A}}(f, g)=\frac{1}{2 \pi \mathrm{i}} \int_{C_{S}} f d g=\operatorname{res}_{P_{+}}(f d g)
$$

(здесь $C_{S}-$ малый контур, окружающий точку $P_{+}$).

Следовательно,

$$
\gamma^{\prime}(L, M)=\alpha \operatorname{res}_{P_{+}}(\operatorname{tr}(L) \cdot \operatorname{tr}(d M))=\alpha \gamma_{2}(L, M)
$$

по определению (3.28).

Для коцикла $\gamma^{\prime \prime}$ на $\overline{\mathfrak{s l}}(n)$ мы используем предложение 4.8 и получаем $\gamma^{\prime \prime}=$ $\beta \gamma_{1, \omega}$. Тем самым мы доказали следующий результат.

ПРЕДЛОЖЕНИЕ 4.10.

$$
\operatorname{dim} \mathrm{H}_{\text {loc, }, \mathscr{L}}(\overline{\mathfrak{g l}}(n), \mathbb{C})=2 .
$$

Базис дается классами когомологий кочиклов $\gamma_{1, \omega} u \gamma_{2}$. Более того, каждый $\mathscr{L}$-инвариантный ограниченный сверху коцикл локален.

\section{5. Единственность класса когомологий для простого случая}

С помощью совершенно другой техники мы покажем в этом разделе, что для простой алгебры Ли пространство локальных классов когомологий самое большее одномерно. Априори мы не будем требовать $\mathscr{L}$-инвариантности. Комбинируя этот результат с результатом предыдущего раздела, состоящим в том, 
что для простой алгебры Ли пространство $\mathscr{L}$-инвариантных локальных когомологических классов одномерно, мы видим, что в простом случае каждый локальный когомологический класс автоматически $\mathscr{L}$-инвариантен. Более того, было показано, что он имеет единственный $\mathscr{L}$-инвариантный представляющий коцикл, кратный $\gamma_{1, \omega}$.

ТеОРема 5.1. Пусть $\mathfrak{g}$ - простая классическая алгебра Ли над $\mathbb{C} u \overline{\mathfrak{g}}-$ ассоциированная алгебра операторов Лакса с ее почти градуировкой. Каждый локальный коцикл на $\overline{\mathfrak{g}}$ с точностью до пропорциональности когомологичен единственному коциклу, ограниченному сверху нулем. В частности, пространство локальных классов когомологий самое большее одномерно, т.е. с точностъю до пропорииональности и эквивалентности есть только один нетривиальный локальный класс когомологий.

ЗАмечАниЕ 5.2. Мы покажем даже следующее. Пусть $\mathfrak{g}-$ простая конечномерная алгебра Ли и $\overline{\mathfrak{g}}-$ любая ассоциированная двухточечная алгебра токов, т. е. алгебра операторов Лакса, алгебра токов Кричевера-Новикова $\mathfrak{g} \otimes \mathscr{A}$ или алгебра петель $\mathfrak{g} \otimes \mathbb{C}\left[z, z^{-1}\right]$. Тогда каждый ограниченный сверху коцикл когомологичен коциклу, который полностью задан своим значением на одной выделенной паре элементов в $\overline{\mathfrak{g}}$ (а именно, значением $\gamma\left(H_{1}^{\alpha}, H_{-1}^{\alpha}\right)$ для одного фиксированного простого корня $\alpha$, см. обозначения ниже). Следовательно, в этих случаях пространства когомологий самое большее одномерны. Кроме структуры $\mathfrak{g}$ мы используем только почти градуированность $\overline{\mathfrak{g}}$ и главные члены, заданные соотношением (5.4).

Для доказательства теоремы 5.1 необходима подготовительная работа.

Сначала напомним следующие факты о генераторах Шевалле алгебры $\mathfrak{g}$. Выберем корневое разложение

$$
\mathfrak{g}=\mathfrak{h} \oplus \bigoplus_{\alpha \in \Delta} \mathfrak{g}^{\alpha}
$$

Как обычно, $\Delta$ обозначает множество всех корней $\alpha \in \mathfrak{h}^{*}$. Далее, пусть $\left\{\alpha_{1}\right.$, $\left.\alpha_{2}, \ldots, \alpha_{p}\right\}$ - базис простых корней $(p=\operatorname{dim} \mathfrak{h})$. По отношению к нему система корней расщепляется на положительные и отрицательные корни, $\Delta_{+}$и $\Delta_{-}$ соответственно. Если $\alpha$ - положительный корень, то $-\alpha-$ отрицательный, и наоборот. Для всех $\alpha \in \Delta$ имеем $\operatorname{dim} \mathfrak{g}^{\alpha}=1$. Можно указать определенные элементы $E^{\alpha} \in \mathfrak{g}^{\alpha}$ и $H^{\alpha} \in \mathfrak{h}$ такие, что для каждого положительного корня $\alpha$

$$
\left[E^{\alpha}, E^{-\alpha}\right]=H^{\alpha}, \quad\left[H^{\alpha}, E^{\alpha}\right]=2 E^{\alpha}, \quad\left[H^{\alpha}, E^{-\alpha}\right]=-2 E^{-\alpha} .
$$

Мы используем также обозначение $H^{i}:=H^{\alpha_{i}}, i=1, \ldots, p$, для элементов, ассоциированных в этом смысле с простыми корнями. Элементы

$$
\left\{E^{\alpha}, \alpha \in \Delta ; H^{i}, 1 \leqslant i \leqslant p\right\}
$$

образуют базис Шевалле (это базис $\mathfrak{g}$ как линейного пространства). 
Обозначим $(\cdot, \cdot)$ скалярное произведение на $\mathfrak{h}^{*}$, индуцированное формой Картана-Киллинга алгебры g. Имеют место следующие соотношения:

$$
\begin{aligned}
{\left[H^{\alpha}, H^{\beta}\right] } & =0, \\
{\left[H^{\alpha}, E^{\beta}\right] } & =2 \frac{(\beta, \alpha)}{(\alpha, \alpha)} E^{\beta}, \\
{\left[H, E^{\alpha}\right] } & =\alpha(H) E^{\alpha}, \quad H \in \mathfrak{h}, \\
{\left[E^{\alpha}, E^{\beta}\right] } & = \begin{cases}H^{\alpha}, & \alpha \in \Delta_{+}, \beta=-\alpha, \\
-H^{\alpha}, & \alpha \in \Delta_{-}, \beta=-\alpha, \\
\pm(r+1) E^{\alpha+\beta}, & \alpha, \beta, \alpha+\beta \in \Delta, \\
0 & \text { в остальных случаях. }\end{cases}
\end{aligned}
$$

Здесь $r$ - наибольшее неотрицательное целое такое, что $\alpha-r \beta$ все еще корень.

Как и в других частях этой статьи, мы обозначаем через $E_{n}^{\alpha}$ и $H_{n}^{\alpha}$ элементы алгебры $\overline{\mathfrak{g}}$ степени $n$, разложения которых в $P^{+}$начинаются с $E^{\alpha} z_{+}^{n}$ и $H^{\alpha} z_{+}^{n}$ соответственно. Следующие элементы образуют базис в $\overline{\mathfrak{g}}$ :

$$
\left\{E_{n}^{\alpha}, \alpha \in \Delta ; H_{n}^{i}, 1 \leqslant i \leqslant p \mid n \in \mathbb{Z}\right\} .
$$

Структурные соотношения, с точностью до членов высшей степени, таковы:

$$
\begin{aligned}
{\left[H_{n}^{\alpha}, H_{m}^{\beta}\right] } & \equiv 0, \\
{\left[H_{n}^{\alpha}, E_{m}^{\beta}\right] } & \equiv 2 \frac{(\beta, \alpha)}{(\alpha, \alpha)} E_{n+m}^{\beta}, \\
{\left[H_{n}, E_{m}^{\alpha}\right] } & \equiv \alpha(H) E_{n+m}^{\alpha}, \quad H \in \mathfrak{h}, \\
{\left[E_{n}^{\alpha}, E_{m}^{\beta}\right] } & \equiv \begin{cases}H_{n+m}^{\alpha}, & \alpha \in \Delta_{+}, \beta=-\alpha, \\
-H_{n+m}^{\alpha}, & \alpha \in \Delta_{-}, \beta=-\alpha, \\
\pm(r+1) E_{n+m}^{\alpha+\beta}, & \alpha, \beta, \alpha+\beta \in \Delta, \\
0 & \text { в остальных случаях. }\end{cases}
\end{aligned}
$$

Значение символа 三 определено в предыдущем разделе (с. 155). В частности, в равенствах (5.4) не выписаны члены степени выше $n+m$. Напомним также, что ввиду почти градуированности существует $K$, не зависящее от $n$ и $m$, такое, что рассматриваемые равенства содержат лишь элементы степени не выше $n+$ $m+K$.

Пусть $\gamma^{\prime}-$ коцикл на $\overline{\mathfrak{g}}$, ограниченный сверху. Для образующих из $\mathfrak{g}$ мы имеем

$$
E^{ \pm \alpha}= \pm \frac{1}{2}\left[H^{\alpha}, E^{ \pm \alpha}\right], \quad H^{i}=\left[E^{\alpha_{i}}, E^{-\alpha_{i}}\right], \quad i=1, \ldots, p .
$$

Следовательно, в $\overline{\mathfrak{g}}$ получаем

$$
\begin{aligned}
E_{n}^{ \pm \alpha} & = \pm \frac{1}{2}\left[H_{0}^{\alpha}, E_{n}^{ \pm \alpha}\right]+Y(n, \alpha), \\
H_{n}^{i} & =\left[E_{0}^{\alpha_{i}}, E_{n}^{-\alpha_{i}}\right]+Z(n, i), \quad i=1, \ldots, p,
\end{aligned}
$$

где $Y(n, \alpha)$ и $Z(n, i)$ являются суммами элементов степеней между $n+m+1$ и $n+m+K$. Зафиксируем число $M \in \mathbb{Z}$ такое, что коцикл $\gamma^{\prime}$ обращается в нуль 
на уровне $M$ и выше. Определим линейное отображение $\Phi: \overline{\mathfrak{g}} \rightarrow \mathbb{C}$ с помощью (нисходящей) индукции по степени базисных элементов (5.3). Положим

$$
\Phi\left(E_{n}^{\alpha}\right)=\Phi\left(H_{n}^{i}\right)=0, \quad \alpha \in \Delta, \quad i=1, \ldots, p, \quad n \geqslant M
$$

Далее определяем индуктивно $\left(\alpha \in \Delta_{+}\right)$

$$
\begin{aligned}
\Phi\left(E_{n}^{ \pm \alpha}\right) & := \pm \frac{1}{2} \gamma^{\prime}\left(H_{0}^{\alpha}, E_{n}^{ \pm a}\right)+\Phi(Y(n, \pm \alpha)), \\
\Phi\left(H_{n}^{i}\right) & :=\gamma^{\prime}\left(E_{0}^{\alpha_{i}}, E_{n}^{-\alpha_{i}}\right)+\Phi(Z(n, i)) .
\end{aligned}
$$

Коцикл $\gamma=\gamma^{\prime}-\delta \Phi$ когомологичен исходному коциклу $\gamma^{\prime}$. Так как коцикл $\gamma^{\prime}$ ограничен сверху и, по определению, $\Phi$ также ограничен сверху, то и коцикл $\gamma$ ограничен сверху.

Согласно конструкции отображения $\Phi$ имеем $\Phi\left(\left[H_{0}^{\alpha}, E_{n}^{ \pm \alpha}\right]\right)=\gamma^{\prime}\left(H_{0}^{\alpha}, E_{n}^{ \pm \alpha}\right)$ и $\Phi\left(\left[E_{0}^{\alpha_{i}}, E_{n}^{-\alpha_{i}}\right]\right)=\gamma^{\prime}\left(E_{0}^{\alpha_{i}}, E_{n}^{-\alpha_{i}}\right)$. Следовательно, справедливо следующее предложение.

ПРеДЛОЖЕНИЕ 5.3. При $\alpha \in \Delta_{+}, i=1, \ldots, p, n \in \mathbb{Z}$ справедливъ равенства

$$
\gamma\left(H_{0}^{\alpha}, E_{n}^{ \pm \alpha}\right)=0, \quad \gamma\left(E_{0}^{\alpha_{i}}, E_{n}^{-\alpha_{i}}\right)=0 .
$$

ОПРЕДЕЛЕНИЕ 5.4. Коцикл $\gamma$ называется нормализованным, если он удовлетворяет условию (5.9).

Таким образом, выше показано, что каждый ограниченный сверху коцикл когомологичен нормализованному, и также ограниченному сверху. Ниже мы предполагаем, что наш коцикл уже нормализован.

ПреДЛОЖЕНИЕ 5.5. Пусть $H$ - произвольный элемент из $\mathfrak{h}$, тогда

$$
\gamma\left(E_{m}^{\alpha}, H_{n}\right) \equiv 0, \quad \alpha \in \Delta, \quad n, m \in \mathbb{Z},
$$

т.е. эти значения являются универсальными выражениями от значений на высших уровнях.

ДокАЗАТЕльство. По определению коцикла

$$
\gamma\left(\left[H_{n}, H_{0}^{\alpha}\right], E_{m}^{\alpha}\right)+\gamma\left(\left[H_{0}^{\alpha}, E_{m}^{\alpha}\right], H_{n}\right)+\gamma\left(\left[E_{m}^{\alpha}, H_{n}\right], H_{0}^{\alpha}\right)=0 .
$$

Коммутатор в первом слагаемом принадлежит высшему уровню. Следовательно, с помощью соотношений (5.4) мы получаем

$$
\alpha\left(H^{\alpha}\right) \gamma\left(E_{m}^{\alpha}, H_{n}\right)+\alpha(H) \gamma\left(E_{m+n}^{\alpha}, H_{0}^{\alpha}\right) \equiv 0 .
$$

Ввиду (5.9) последний член обращается в нуль. Так как $\alpha\left(H^{\alpha}\right) \neq 0$, получаем требуемое утверждение.

ПрЕДЛОЖЕНИЕ 5.6. Пусть $\alpha$ и $\beta$-корни такие, что $\beta \neq-\alpha$, тогда

$$
\gamma\left(E_{m}^{\alpha}, E_{n}^{\beta}\right) \equiv 0, \quad n, m \in \mathbb{Z},
$$

т.е. они являются (универсальными) выражениями от значений высшего уровня. 
ДокАЗАТЕЛЬство. Пусть $H$ - произвольный элемент $\mathfrak{h}$. Снова по определению коцикла

$$
\gamma\left(\left[E_{m}^{\alpha}, H_{0}\right], E_{n}^{\beta}\right)+\gamma\left(\left[H_{0}, E_{n}^{\beta}\right], E_{m}^{\alpha}\right)+\gamma\left(\left[E_{n}^{\beta}, E_{m}^{\alpha}\right], H_{0}\right)=0 .
$$

Здесь третий член имеет высший уровень. Если $\alpha+\beta \in \Delta$, это следует из (5.10). Если же $\alpha+\beta \notin \Delta$, то $\left[E^{\alpha}, E^{\beta}\right]=0$ и степень $\left[E_{n}^{\alpha}, E_{m}^{\beta}\right]$ выше, чем $m+n$.

Для первых двух членов мы находим (с помощью (5.4))

$$
(\alpha+\beta)(H) \gamma\left(E_{n}^{\beta}, E_{m}^{\alpha}\right) \equiv 0 .
$$

Поскольку мы можем выбрать $H$ так, что $(\alpha+\beta)(H) \neq 0$, мы получаем требуемое утверждение.

Из определения коцикла следует, что

$$
\gamma\left(\left[E_{0}^{\alpha}, E_{n}^{-\alpha}\right], H_{m}^{\beta}\right)+\gamma\left(\left[E_{n}^{-\alpha}, H_{m}^{\beta}\right], E_{0}^{\alpha}\right)+\gamma\left(\left[H_{m}^{\beta}, E_{0}^{\alpha}\right], E_{n}^{-\alpha}\right)=0 .
$$

Пользуясь соотношением (5.4) и игнорируя высшие уровни, для положительных корней $\alpha$ и $\beta$ получаем

$$
\gamma\left(H_{n}^{\alpha}, H_{m}^{\beta}\right)+2 \frac{(\alpha, \beta)}{(\alpha, \alpha)}\left(\gamma\left(E_{n+m}^{-\alpha}, E_{0}^{\alpha}\right)+\gamma\left(E_{m}^{\alpha}, E_{n}^{-\alpha}\right)\right) \equiv 0 .
$$

ПрЕДЛОЖЕНИЕ 5.7. Пусть $\alpha$ - простой коренъ, тогда

$$
\gamma\left(E_{n}^{-\alpha}, E_{m}^{\alpha}\right) \equiv \frac{1}{2} \gamma\left(H_{n}^{\alpha}, H_{m}^{\alpha}\right)
$$

ДоказАтельство. Возьмем в (5.17) $\alpha$ и $\beta$ равными одному и тому же простому корню. Ввиду предложения 5.3 выполнено равенство $\gamma\left(E_{n+m}^{-\alpha}, E_{0}^{\alpha}\right)=0$, что и доказывает требуемое утверждение.

Комбинируя (5.17) и предложение 5.7, мы получаем для простого корня $\alpha$ и произвольного корня $\beta$

$$
\gamma\left(H_{n}^{\alpha}, H_{m}^{\beta}\right) \equiv \frac{(\alpha, \beta)}{(\alpha, \alpha)} \gamma\left(H_{n}^{\alpha}, H_{m}^{\alpha}\right) .
$$

ПреДЛОЖЕНИЕ 5.8. Пусть $\alpha$ - положительный корень, $\alpha_{1}$ - простой корень, причем $\alpha+\alpha_{1}$ снова корень, тогда

$$
\gamma\left(E_{m}^{\alpha+\alpha_{1}}, E_{n}^{-\left(\alpha+\alpha_{1}\right)}\right) \equiv s_{\alpha, \alpha_{1}} \gamma\left(E_{m}^{\alpha}, E_{n}^{-\alpha}\right),
$$

где $s_{\alpha, \alpha_{1}} \neq 0$ - константа.

ДокАЗАТЕЛЬСТво. По определению коцикла

$$
\gamma\left(\left[E_{m}^{\alpha+\alpha_{1}}, E_{0}^{-\alpha_{1}}\right], E_{n}^{-\alpha}\right)+\gamma\left(\left[E_{0}^{-\alpha_{1}}, E_{n}^{-\alpha}\right], E_{m}^{\alpha+\alpha_{1}}\right)+\gamma\left(\left[E_{n}^{-\alpha}, E_{m}^{\alpha+\alpha_{1}}\right], E_{0}^{-\alpha_{1}}\right)=0 .
$$

Так как $\alpha_{1}$ является простым корнем, мы можем применить предложение 5.3 и увидеть, что третий член имеет высший уровень. Для первых двух слагаемых воспользуемся одним из соотношений (5.4), именно, соотношением, содержащим $r$. Так как в (5.4) $r+1 \neq 0$, получаем требуемое.

ПреДЛОЖЕНИЕ 5.9. Пусть $\alpha$ и $\beta$-два простых корня. Тогда

$$
\gamma\left(H_{n}^{\alpha}, H_{m}^{\alpha}\right) \equiv \frac{(\alpha, \alpha)}{(\beta, \beta)} \gamma\left(H_{n}^{\beta}, H_{m}^{\beta}\right) .
$$


ДокАЗАТЕЛЬСтво. Ввиду (5.19)

$$
\gamma\left(H_{n}^{\alpha}, H_{m}^{\beta}\right) \equiv \frac{(\alpha, \beta)}{(\alpha, \alpha)} \gamma\left(H_{n}^{\alpha}, H_{m}^{\alpha}\right)
$$

и аналогично

$$
\gamma\left(H_{m}^{\beta}, H_{n}^{\alpha}\right) \equiv \frac{(\beta, \alpha)}{(\beta, \beta)} \gamma\left(H_{m}^{\beta}, H_{n}^{\beta}\right) .
$$

Так как $\gamma$ - кососимметричная, а $(\cdot, \cdot)$ - симметричная форма, находим

$$
\frac{(\alpha, \beta)}{(\alpha, \alpha)} \gamma\left(H_{n}^{\alpha}, H_{m}^{\alpha}\right) \equiv \frac{(\alpha, \beta)}{(\beta, \beta)} \gamma\left(H_{n}^{\beta}, H_{m}^{\beta}\right) .
$$

Если $(\alpha, \beta) \neq 0$, мы непосредственно получаем (5.22). Если нет, то благодаря неприводимости системы корней мы всегда можем найти цепочку простых корней $\alpha^{(j)}, j=0, \ldots, k$, такую, что $\alpha^{(0)}=\alpha, \alpha^{(k)}=\beta$ и $\left(\alpha^{(j)}, \alpha^{(j+1)}\right) \neq 0$. Применяя соотношение (5.22), уже доказанное в этой ситуации, вдоль цепочки, получим требуемое.

ПРЕДЛОЖЕНИЕ 5.10. Пусть $\alpha_{1}-\oint$ бисированный простой коренъ, $\alpha-$ произвольный положительный корень, тогда

$$
\gamma\left(E_{m}^{\alpha}, E_{n}^{-\alpha}\right) \equiv s_{\alpha, \alpha_{1}} \gamma\left(E_{m}^{\alpha_{1}}, E_{n}^{-\alpha_{1}}\right) \equiv t_{\alpha, \alpha_{1}} \gamma\left(H_{m}^{\alpha_{1}}, H_{n}^{\alpha_{1}}\right),
$$

где $s_{\alpha, \alpha_{1}} \neq 0, t_{\alpha, \alpha_{1}} \neq 0$ - постоянные.

ДокАЗАТЕльство. Так как $\alpha$ - положительный корень, он является нетривиальной суммой простых корней. Пусть $\alpha_{2}$ - один из них. Многократное применение предложения 5.8 показывает, что значение $\gamma\left(E_{m}^{\alpha}, E_{n}^{-\alpha}\right)$ может быть сведено к $\gamma\left(E_{m}^{\alpha_{2}}, E_{n}^{-\alpha_{2}}\right)$. Комбинация предложений 5.7 и 5.9 дает (5.24).

ПРЕДЛОЖЕНИЕ 5.11. Пусть $\alpha_{1}$ - биксированный простой коренъ, а $\alpha$ и $\beta$ произвольные корни, тогда

$$
\gamma\left(H_{n}^{\alpha}, H_{m}^{\beta}\right) \equiv s_{\alpha, \beta} \gamma\left(H_{n}^{\alpha_{1}}, H_{m}^{\alpha_{1}}\right), \quad \text { əде } s_{\alpha, \beta} \in \mathbb{C} .
$$

ДокАзАТЕльство. Так как $H^{\alpha_{i}}, i=1, \ldots, p$, образуют базис картановской подалгебры $\mathfrak{g}$, каждый элемент $H^{\alpha}$ является линейной их комбинацией. Это продолжается и на элементы $H_{n}^{\alpha}$. Предложение вытекает из билинейности коцикла, предложения 5.9 и соотношения (5.19).

Суммируем результаты, полученные в предложениях 5.3, 5.6, 5.10 и 5.11.

ПРЕДЛОЖЕНИЕ 5.12. Пусть $\alpha_{1}-$ фиксированный простой коренъ, а $\gamma-$ определенный выше кочикл, тогда для всех $n, m \in \mathbb{Z}$

$$
\begin{aligned}
\gamma\left(E_{m}^{\alpha}, H_{n}\right) & \equiv 0, \quad H \in \mathfrak{h}, \quad \alpha \in \Delta, \\
\gamma\left(E_{m}^{\alpha}, E_{n}^{\beta}\right) & \equiv 0, \quad \alpha, \beta \in \Delta, \quad \beta \neq-\alpha, \\
\gamma\left(E_{m}^{\alpha}, E_{n}^{-\alpha}\right) & \equiv s \gamma\left(H_{m}^{\alpha_{1}}, H_{n}^{\alpha_{1}}\right), \quad \alpha \in \Delta, \\
\gamma\left(H_{m}^{\alpha}, H_{n}^{\beta}\right) & \equiv t \gamma\left(H_{m}^{\alpha_{1}}, H_{n}^{\alpha_{1}}\right), \quad \alpha, \beta \in \Delta_{+},
\end{aligned}
$$

əде $s, t \in \mathbb{C}$. 
Пусть $\alpha$ - простой корень; рассмотрим соотношение

$$
\gamma\left(H_{m}^{\alpha},\left[E_{n}^{\alpha}, E_{k}^{-\alpha}\right]\right)+\gamma\left(E_{n}^{\alpha},\left[E_{k}^{-\alpha}, H_{m}^{\alpha}\right]\right)+\gamma\left(E_{k}^{-\alpha},\left[H_{m}^{\alpha}, E_{n}^{\alpha}\right]\right)=0 .
$$

Используя (5.4), получим

$$
\gamma\left(H_{m}^{\alpha}, H_{n+k}^{\alpha}\right)+\gamma\left(E_{n}^{\alpha}, 2 E_{k+m}^{-\alpha}\right)+\gamma\left(E_{k}^{-\alpha}, 2 E_{m+n}^{\alpha}\right) \equiv 0 .
$$

Поскольку корень простой, мы можем воспользоваться предложением 5.7 и получить важное соотношение

$$
\gamma\left(H_{m}^{\alpha}, H_{n+k}^{\alpha}\right)+\gamma\left(H_{n}^{\alpha}, H_{k+m}^{\alpha}\right)+\gamma\left(H_{k}^{\alpha}, H_{m+n}^{\alpha}\right) \equiv 0 .
$$

ПРЕДЛОЖЕНИЕ 5.13. Пусть $\alpha$ - простой коренъ, тогда

$$
\gamma\left(H_{n}^{\alpha}, H_{0}^{\alpha}\right) \equiv 0
$$

u

$$
\gamma\left(H_{n+1}^{\alpha}, H_{l-(n+1)}^{\alpha}\right) \equiv \gamma\left(H_{n-1}^{\alpha}, H_{l-(n-1)}^{\alpha}\right)+2 \gamma\left(H_{1}^{\alpha}, H_{l-1}^{\alpha}\right) .
$$

ДокАЗАТЕЛЬСтво. Возьмем в (5.29) $m=k=0$, тогда (5.30) следует из антисимметричности. Полагая в (5.29) $m=-1$ и $k=l-n+1$, получим

$$
\gamma\left(H_{-1}^{\alpha}, H_{l+1}^{\alpha}\right)+\gamma\left(H_{n}^{\alpha}, H_{l-n}^{\alpha}\right)+\gamma\left(H_{l-(n-1)}^{\alpha}, H_{n-1}^{\alpha}\right) \equiv 0 .
$$

При $m=1$ и $k=l-n-1$ мы получаем

$$
\gamma\left(H_{1}^{\alpha}, H_{l-1}^{\alpha}\right)+\gamma\left(H_{n}^{\alpha}, H_{l-n}^{\alpha}\right)+\gamma\left(H_{l-(n+1)}^{\alpha}, H_{n+1}^{\alpha}\right) \equiv 0 .
$$

Вычитая (5.32) из (5.33), получим

$$
\gamma\left(H_{l-(n+1)}^{\alpha}, H_{n+1}^{\alpha}\right) \equiv \gamma\left(H_{l-(n-1)}^{\alpha}, H_{n-1}^{\alpha}\right)-\gamma\left(H_{1}^{\alpha}, H_{l-1}^{\alpha}\right)+\gamma\left(H_{-1}^{\alpha}, H_{l+1}^{\alpha}\right) .
$$

Полагая в (5.29) $n=-m$ и $k=l$, получаем

$$
\gamma\left(H_{-n}^{\alpha}, H_{n+l}^{\alpha}\right)+\gamma\left(H_{n}^{\alpha}, H_{l-n}^{\alpha}\right)+\gamma\left(H_{l}^{\alpha}, H_{0}^{\alpha}\right) \equiv 0 .
$$

Ввиду (5.30) последний член не вносит никакого вклада. Следовательно,

$$
\gamma\left(H_{n}^{\alpha}, H_{l-n}^{\alpha}\right) \equiv-\gamma\left(H_{-n}^{\alpha}, H_{l+n}^{\alpha}\right) .
$$

Если подставить (5.36) в (5.34) и воспользоваться антисимметричностью, получим (5.31).

ПреДЛОЖЕНИЕ 5.14. Пусть $\alpha$ - простой коренъ. На уровне $l=0$ значения кочикла даются соотношениями

$$
\gamma\left(H_{n}^{\alpha}, H_{-n}^{\alpha}\right) \equiv n \gamma\left(H_{1}^{\alpha}, H_{-1}^{\alpha}\right), \quad \gamma\left(H_{0}^{\alpha}, H_{0}^{\alpha}\right)=0 .
$$

ДокАЗАТЕЛЬСтво. Если положить в (5.31) $l=0$, мы получим соотношение

$$
\gamma\left(H_{n+1}^{\alpha}, H_{-(n+1)}^{\alpha}\right) \equiv \gamma\left(H_{n-1}^{\alpha}, H_{-(n-1)}^{\alpha}\right)+2 \gamma\left(H_{1}^{\alpha}, H_{-1}^{\alpha}\right),
$$

которое приводит к нужному выражению. 
ПРЕДЛОЖЕНИЕ 5.15. Для простого корня $\alpha$ и уровня $l \neq 0$ имеем

$$
\gamma\left(H_{n}^{\alpha}, H_{l-n}^{\alpha}\right) \equiv 0 .
$$

ДоказАтельство. Сначала возьмем $l>0$. Применяя рекурсию (5.31) и соотношение (5.30), мы видим, что утверждение окажется справедливым, если проверить его для $\gamma\left(H_{1}^{\alpha}, H_{l-1}^{\alpha}\right)$. Для $l=1$, применяя (5.30), получаем $\gamma\left(H_{1}^{\alpha}, H_{0}^{\alpha}\right) \equiv 0$. Для $l=2$, ввиду антисимметрии, $\gamma\left(H_{1}^{\alpha}, H_{1}^{\alpha}\right)=0$. Пусть $l>2$. Положим в (5.29) $k=l-r-1, n=1$ и $m=r$ :

$$
\gamma\left(H_{1}^{\alpha}, H_{l-1}^{\alpha}\right)+\gamma\left(H_{r}^{\alpha}, H_{l-r}^{\alpha}\right)-\gamma\left(H_{r+1}^{\alpha}, H_{l-(r+1)}^{\alpha}\right) \equiv 0 .
$$

Положим $m=(l-2) / 2$ для четных $l$ и $m=(l-1) / 2$ для нечетных. Когда $r$ пробегает значения $1,2, \ldots, m$, мы получаем $m$ соотношений. Первое всегда имеет вид

$$
2 \gamma\left(H_{1}^{\alpha}, H_{l-1}^{\alpha}\right)-\gamma\left(H_{2}^{\alpha}, H_{l-2}^{\alpha}\right) \equiv 0 .
$$

Структура последнего соотношения зависит от четности $l$. Для четных $l$ и $r=$ $m$ последний член в $(5.40)$ есть $\gamma\left(H_{l / 2}^{\alpha}, H_{l / 2}^{\alpha}\right)$, что равно нулю. Для нечетных $l$ последний член в (5.40) совпадает со вторым. Следовательно,

$$
\gamma\left(H_{1}^{\alpha}, H_{l-1}^{\alpha}\right)+2 \gamma\left(H_{(l-1) / 2}^{\alpha}, H_{(l+1) / 2}^{\alpha}\right) \equiv 0 .
$$

В этом случае мы делим полученное на 2. Суммируя все полученные соотношения, получаем

$$
(m+\epsilon) \gamma\left(H_{1}^{\alpha}, H_{l-1}^{\alpha}\right) \equiv 0,
$$

где $\epsilon=1$ для четных $l$ и $\epsilon=1 / 2$ для нечетных. Так как в любом случае $m+\epsilon>0$, это доказывает утверждение.

Для $l<0$ нетрудно видеть, что утверждение справедливо, если оно верно для $\gamma\left(H_{-1}^{\alpha}, H_{l+1}^{\alpha}\right)$. Аргументы те же, что и выше. Для $l=-1,-2$ это получается немедленно. Для $l<-2$ мы подставляем в (5.29) $k=l-r+1, n=-1$ и $m=r$ и получаем

$$
\gamma\left(H_{-1}^{\alpha}, H_{l+1}^{\alpha}\right)+\gamma\left(H_{r}^{\alpha}, H_{l-r}^{\alpha}\right)-\gamma\left(H_{r-1}^{\alpha}, H_{l-(r-1)}^{\alpha}\right) \equiv 0 .
$$

Положим $m=(-l-2) / 2$ для четных $l$ и $m=(-l-1) / 2$ для нечетных и рассмотрим соотношения (5.44) для $r=-1,-2, \ldots,-m$. Они имеют структуру, аналогичную структуре при $l>0$, и мы можем получить утверждение об $\gamma\left(H_{-1}^{\alpha}, H_{l+1}^{\alpha}\right)$ с помощью их суммирования.

ДОКАЗАТЕЛЬСТВО ТЕОРЕМЫ 5.1. Добавляя подходящую кограницу, мы можем заменить данный коцикл $\gamma$ нормализованным (см. определение 5.4). С помощью доказанных выше утверждений мы показали, что значения коцикла на уровне $l$ линейно выражаются через значения на уровнях выше $l$ и значения вида $\gamma\left(H_{n}^{\alpha}, H_{l-n}^{\alpha}\right)$. При $l>0$ ввиду предложения 5.15 эти значения также могут быть выражены через высшие уровни. Следовательно, индуктивно, начиная с верхней границы коцикла, мы получаем, что верхней границей уровня ненулевых значений коцикла является нуль. Также мы получаем, что значения на 
уровнях $l<0$ определяются значениями на нулевом уровне. Остается разобраться с нулевым уровнем. Ввиду предложений 5.12, 5.14 и 5.15 все зависит лишь от значения $\gamma\left(H_{1}^{\alpha}, H_{-1}^{\alpha}\right)$ для одного-единственного простого корня. Это доказывает теорему.

ПРЕДЛОЖЕНИЕ 5.16. Если нормализованный коцикл является кограницей, то он тождественно равен нулю.

ДокАзАТЕльство. Как объяснялось выше, нормализованный коцикл полностью задается значением $\gamma\left(H_{1}^{\alpha}, H_{-1}^{\alpha}\right)$. Но

$$
H_{(1)}^{\alpha}:=H_{0}^{\alpha} A_{1} \equiv H_{1}^{\alpha} \quad \text { и } \quad H_{(-1)}^{\alpha}:=H_{0}^{\alpha} A_{-1} \equiv H_{-1}^{\alpha} .
$$

Следовательно,

$$
\left[H_{(1)}^{\alpha}, H_{(-1)}^{\alpha}\right]=\left[H_{0}^{\alpha}, H_{0}^{\alpha}\right] A_{1} A_{-1}=0 .
$$

Так как коцикл обращается в нуль на положительных уровнях и так как $\gamma=\delta \phi-$ кограница, мы получаем

$$
\gamma\left(H_{1}^{\alpha}, H_{-1}^{\alpha}\right)=\gamma\left(H_{(1)}^{\alpha}, H_{(-1)}^{\alpha}\right)=\phi\left(\left[H_{(1)}^{\alpha}, H_{(-1)}^{\alpha}\right]\right)=\phi(0)=0 .
$$

Следовательно, и все значения коцикла нулевые, что и утверждается.

ЗАмечАнИЕ 5.17. В классическом случае $\overline{\mathfrak{g}}=\mathfrak{g} \otimes \mathbb{C}\left[z, z^{-1}\right]$, когда алгебра градуирована и в (5.6) нет членов высшего порядка, мы могли бы стартовать с произвольного коцикла, не обязательно ограниченного. Мы могли бы взять в качестве кограницы (5.8). Все символы 三 заменились бы знаком равенства, не было бы никаких вкладов высшего уровня, а результат остался бы тем же самым. В этом весьма специальном случае представленное выше доказательство упрощается и становится аналогичным доказательству Гарланда [14].

ЗАмЕчАНИЕ 5.18. Более внимательный анализ аргументов, использованных в разделах 4 и 5, показывает, что мы используем только: 1) свойство почти градуированности алгебры $\overline{\mathfrak{g}}, 2)$ существование связности $\omega$, голоморфной в $P_{+}$, возможно имеющей полюс в $P_{-}$и точках слабых особенностей, так что $\overline{\mathfrak{g}}$ становится модулем над алгеброй Ли $\mathscr{L}$ по отношению к этой связности, и 3 ) локальность коцикла (3.5) по отношению к почти градуировке. Уже этих условий достаточно для выполнения предложения 2.11 и справедливости всех остальных аргументов при любом подходящем определении алгебры $\overline{\mathfrak{g}}$, связанной с простой алгеброй Ли g.

\section{Приложение А. Вычисления для $\overline{\mathfrak{s o}}(n)$ и $\overline{\mathfrak{s p}}(2 n)$}

В этом приложении мы доказываем предложение 2.9 для $\overline{\mathfrak{g}}=\overline{\mathfrak{s o}}(n)$ и $\overline{\mathfrak{g}}=$ $\overline{\mathfrak{s p}}(2 n)$. На самом деле, остается только показать, что для $L \in \overline{\mathfrak{g}}$ мы имеем $\nabla_{e}^{(\omega)} L \in \overline{\mathfrak{g}}$. Точнее, надо проверить, выполняются ли условия в точках $\gamma_{s}$ с $\alpha_{s} \neq 0$. Для упрощения обозначений мы опускаем индекс $s$ при $z_{s}$ и относящихся к этой точке величинах.

A.1. Случай $\overline{\mathfrak{g}}=\overline{\mathfrak{s o}}(n)$. Элемент $L \in \overline{\mathfrak{s o}}(n)$ в точках слабых особенностей дается разложением (2.4) и условиями (2.8). Далее, пусть $\omega-$ связность, удовлетворяющая соотношениям (2.32). Первый член в разложении связности, 
примененный к $L$, дает

$$
\frac{d L}{d z}=\frac{-L_{-1}}{z^{2}}+L_{1}+\sum_{k \geqslant 1}(k+1) L_{k+1} z^{k} .
$$

Рассмотрим разложение второго члена $[\omega, L]$.

Член порядка -2 имеет матричный коэффициент

$$
\left[\omega_{-1}, L_{-1}\right]=\left[\alpha \widetilde{\beta}^{t}-\widetilde{\beta} \alpha^{t}, \alpha \beta^{t}-\beta \alpha^{t}\right]
$$

Применяя соотношения

$$
\widetilde{\beta}^{t} \alpha=\alpha^{t} \widetilde{\beta}=1, \quad \alpha^{t} \alpha=0, \quad \beta^{t} \alpha=\alpha^{t} \beta=0, \quad \epsilon:=\widetilde{\beta}^{t} \beta,
$$

находим

$$
\begin{array}{llrl}
{\left[\alpha \widetilde{\beta}^{t}, \alpha \beta^{t}\right]} & =\alpha \beta^{t}, & & {\left[\alpha \widetilde{\beta}^{t}, \beta \alpha^{t}\right]=\epsilon \alpha \alpha^{t},} \\
{\left[\widetilde{\beta} \alpha^{t}, \alpha \beta^{t}\right]} & =-\epsilon \alpha \alpha^{t}, & & {\left[\widetilde{\beta} \alpha^{t}, \beta \alpha^{t}\right]=-\beta \alpha^{t} .}
\end{array}
$$

Следовательно,

$$
\left[\omega_{-1}, L_{-1}\right]=\alpha \beta^{t}-\beta \alpha^{t}=L_{-1},
$$

и член порядка -2 аннулируется.

Член порядка -1: здесь нужно показать, что матричный коэффициент записывается в виде $\alpha \widehat{\beta}^{t}-\widehat{\beta} \alpha^{t}$, где $\widehat{\beta}$ удовлетворяет условию $\widehat{\beta}^{t} \alpha=0$. Имеем

$$
\begin{aligned}
{\left[\omega_{-1}, L_{0}\right] } & =\left[\alpha \widetilde{\beta}^{t}, L_{0}\right]-\left[\widetilde{\beta} \alpha^{t}, L_{0}\right] \\
& =\alpha \widetilde{\beta}^{t} L_{0}-L_{0} \alpha \widetilde{\beta}^{t}-\widetilde{\beta} \alpha^{t} L_{0}+L_{0} \widetilde{\beta} \alpha^{t} \\
& =\alpha\left(\widetilde{\beta}^{t} L_{0}-\varkappa \widetilde{\beta}^{t}\right)+\left(\varkappa \widetilde{\beta}+L_{0} \widetilde{\beta}\right) \alpha^{t}, \\
{\left[L_{-1}, \omega_{0}\right] } & =\alpha\left(\beta^{t} \omega_{0}-\tilde{\varkappa} \beta^{t}\right)+\left(\widetilde{\varkappa} \beta+\omega_{0} \beta\right) \alpha^{t} .
\end{aligned}
$$

Если мы положим

$$
\widehat{\beta}=-\left(\varkappa \widetilde{\beta}+\tilde{\varkappa} \beta+L_{0} \widetilde{\beta}+\omega_{0} \beta\right)
$$

то получим

$$
\left[\omega_{-1}, L_{0}\right]+\left[L_{-1}, \omega_{0}\right]=\alpha \widehat{\beta}^{t}-\widehat{\beta} \alpha^{t}
$$

Далее,

$$
\widehat{\beta}^{t} \alpha=\widetilde{\beta}^{t} L_{0} \alpha+\beta^{t} \omega_{0} \alpha-\varkappa \widetilde{\beta}^{t} \alpha-\tilde{\varkappa} \beta^{t} \alpha=0,
$$

так как $\alpha$ и $\omega_{0}$ являются собственными векторами $L_{0}$ с собственными значениями $\varkappa$ и $\tilde{\varkappa}$ соответственно.

Нулевой порядок: здесь надо показать, что существует $\widehat{\varkappa} \in \mathbb{C}$ такое, что

$$
\left(\left[\omega_{-1}, L_{1}\right]+\left[\omega_{0}, L_{0}\right]+\left[\omega_{1}, L_{-1}\right]+L_{1}\right) \alpha=\widehat{\varkappa} \alpha .
$$

Второе слагаемое обращается в нуль. Далее,

$$
\begin{aligned}
{\left[\omega_{-1}, L_{1}\right] \alpha } & =\alpha \widetilde{\beta}^{t} L_{1} \alpha-\widetilde{\beta} \alpha^{t} L_{1} \alpha-L_{1} \alpha \widetilde{\beta}^{t} \alpha+L_{1} \widetilde{\beta} \alpha^{t} \alpha \\
& =-L_{1} \alpha+\mu \alpha,
\end{aligned}
$$


где $\mu=\widetilde{\beta}^{t} L_{1} \alpha$. Заметим, что $\alpha^{t} L_{1} \alpha=0$, так как $L_{1}-$ кососимметрическая матрица. Последнее слагаемое также обращается в нуль, поскольку $\alpha^{t} \alpha=0$. Также

$$
\left[\omega_{-1}, L_{1}\right] \alpha=\mu^{\prime} \alpha
$$

где $\mu^{\prime}=-\beta^{t} \omega_{1} \alpha$. Следовательно, мы получаем требуемое свойство.

А.2. Случай $\overline{\mathfrak{g}}=\overline{\mathfrak{s p}}(2 n)$. Пусть $\omega$ - форма связности, удовлетворяющая (2.30) и (2.33). Для удобства читателя мы повторим здесь список ее свойств (см. п. 2.1):

$$
\begin{gathered}
\omega_{-1}=\left(\alpha \widetilde{\beta}^{t}+\widetilde{\beta} \alpha^{t}\right) \sigma, \\
\widetilde{\beta}^{t} \sigma \alpha=1, \\
\omega_{0} \alpha=\tilde{\varkappa} \alpha, \\
\alpha^{t} \sigma \omega_{1} \alpha=0 .
\end{gathered}
$$

Соответствующие условия для $L \in \overline{\mathfrak{s p}}(2 n)$ таковы:

$$
\begin{gathered}
L_{-2}=\nu \alpha \alpha^{t}, \quad L_{-1}=\left(\alpha \beta^{t}+\beta \alpha^{t}\right) \sigma, \\
\beta^{t} \sigma \alpha=0, \\
L_{0} \alpha=\varkappa \alpha, \\
\alpha^{t} \sigma L_{1} \alpha=0 .
\end{gathered}
$$

Мы имеем

$$
\begin{gathered}
\frac{d L}{d z}=-2 \nu \frac{\alpha \alpha^{t} \sigma}{z^{3}}-\frac{\left(\alpha \beta^{t}+\beta \alpha^{t}\right) \sigma}{z^{2}}+L_{1}+2 L_{2} z+\cdots, \\
{[\omega, L]=\frac{\left[\omega_{-1}, L_{-2}\right]}{z^{3}}+\frac{\left[\omega_{-1}, L_{-1}\right]+\left[\omega_{0}, L_{-2}\right]}{z^{2}}} \\
+\frac{\left[\omega_{-1}, L_{0}\right]+\left[\omega_{0}, L_{-1}\right]+\left[\omega_{1}, L_{-2}\right]}{z}+\cdots .
\end{gathered}
$$

Пусть

$$
L^{\prime} d z=\nabla^{(\omega)} L=\left(\frac{d L}{d z}+[\omega, L]\right) d z .
$$

Вычислим матричные коэффициенты разложения элемента $L^{\prime}$.

Член порядка -3:

$$
\begin{aligned}
L_{-3}^{\prime} & =-2 \nu \alpha \alpha^{t} \sigma+\left[\left(\alpha \widetilde{\beta}^{t}+\widetilde{\beta} \alpha^{t}\right) \sigma, \nu \alpha \alpha^{t} \sigma\right] \\
& =-2 \nu \alpha \alpha^{t} \sigma+\nu \alpha\left(\widetilde{\beta}^{t} \sigma \alpha\right) \alpha^{t} \sigma-\nu \alpha\left(\alpha^{t} \sigma \widetilde{\beta}\right) \alpha^{t} \sigma .
\end{aligned}
$$

Ввиду (А.2) и антисимметричности $\sigma$ мы получаем $L_{-3}=0$.

Член порядка -2:

$$
\begin{aligned}
L_{-2}^{\prime}= & -\left(\alpha \beta^{t}+\beta \alpha^{t}\right) \sigma+\left[\left(\alpha \widetilde{\beta}^{t}+\widetilde{\beta} \alpha^{t}\right) \sigma,\left(\alpha \beta^{t}+\beta \alpha^{t}\right) \sigma\right]+\nu\left[\omega_{0}, \alpha \alpha^{t} \sigma\right] \\
= & -\left(\alpha \beta^{t}+\beta \alpha^{t}\right) \sigma+\alpha\left(\widetilde{\beta}^{t} \sigma \alpha\right) \beta^{t} \sigma+\alpha\left(\widetilde{\beta}^{t} \sigma \beta\right) \alpha^{t} \sigma \\
& -\alpha\left(\beta^{t} \sigma \widetilde{\beta}\right) \alpha^{t} \sigma-\beta\left(\alpha^{t} \sigma \widetilde{\beta}\right) \alpha^{t} \sigma+2 \nu \widetilde{\varkappa} \alpha \alpha^{t} \\
= & 2\left(\widetilde{\beta}^{t} \sigma \beta+\nu \widetilde{\varkappa}\right) \alpha \alpha^{t} \sigma .
\end{aligned}
$$


Следовательно, $L_{-2}^{\prime}$ имеет требуемую форму (А.5).

Член порядка -1:

$$
\begin{aligned}
L_{-1}^{\prime}= & {\left[\left(\alpha \widetilde{\beta}^{t}+\widetilde{\beta} \alpha^{t}\right) \sigma, L_{0}\right]+\left[\omega_{0},\left(\alpha \beta^{t}+\beta \alpha^{t}\right) \sigma\right]+\left[\omega_{1}, \nu \alpha \alpha^{t} \sigma\right] } \\
= & \left.\alpha \widetilde{\beta}^{t} \sigma, L_{0}\right]+\left[\widetilde{\beta} \alpha^{t} \sigma, L_{0}\right]+\left[\omega_{0}, \alpha \beta^{t} \sigma\right]+\left[\omega_{0}, \beta \alpha^{t} \sigma\right]+\left[\omega_{1}, \nu \alpha \alpha^{t} \sigma\right] \\
=\alpha & \left(\widetilde{\beta}^{t} \sigma L_{0}\right)-\varkappa \alpha \widetilde{\beta}^{t} \sigma-\varkappa \widetilde{\beta} \alpha^{t} \sigma-L_{0} \widetilde{\beta} \alpha^{t} \sigma \\
& \quad+\widetilde{\varkappa} \alpha \beta^{t} \sigma-\alpha\left(\beta^{t} \sigma \omega_{0}\right)+\omega_{0} \beta \alpha^{t} \sigma+\widetilde{\varkappa} \beta \alpha^{t} \sigma \\
& \quad+\nu\left(\omega_{1} \alpha\right) \alpha^{t} \sigma-\nu \alpha \alpha^{t} \sigma \omega_{1} \\
=\alpha & \left(\widetilde{\beta}^{t} \sigma L_{0}\right)-\varkappa \alpha \widetilde{\beta}^{t} \sigma+\widetilde{\varkappa} \alpha \beta^{t} \sigma-\alpha\left(\beta^{t} \sigma \omega_{0}\right)-\nu \alpha \alpha^{t} \sigma \omega_{1} \\
& \quad-\varkappa \widetilde{\beta} \alpha^{t} \sigma-L_{0} \widetilde{\beta} \alpha^{t} \sigma+\omega_{0} \beta \alpha^{t} \sigma+\widetilde{\varkappa} \beta \alpha^{t} \sigma+\nu\left(\omega_{1} \alpha\right) \alpha^{t} \sigma \\
=\alpha & \left(\widetilde{\beta}^{t} \sigma L_{0}-\varkappa \widetilde{\beta}^{t} \sigma+\widetilde{\varkappa} \beta^{t} \sigma-\beta^{t} \sigma \omega_{0}-\nu \alpha^{t} \sigma \omega_{1}\right) \\
& \quad+\left(-\varkappa \widetilde{\beta}-L_{0} \widetilde{\beta}+\omega_{0} \beta+\widetilde{\varkappa} \beta+\nu \omega_{1} \alpha\right) \alpha^{t} \sigma .
\end{aligned}
$$

Обозначим вторую скобку в последнем выражении через $\beta^{\prime}$. Тогда, ввиду соотношений симплектичности $\omega_{1}^{t}=-\sigma \omega_{1} \sigma^{-1}$ и соответствующих соотношений для $\omega_{0}$ и $L_{0}$, мы находим, что первая скобка равна $\beta^{\prime t} \sigma$, следовательно,

$$
L_{-1}^{\prime}=\left(\alpha \beta^{\prime t}+\beta^{\prime} \alpha^{t}\right) \sigma,
$$

как и требуется соотношением (А.5).

Остается показать, что $\beta^{\prime t} \sigma \alpha=0$. Выражение для $\beta^{\prime t} \sigma$ в точности дается только что упомянутой первой скобкой. Имеем

$$
\begin{aligned}
\beta^{t} \sigma \alpha & =\left(\widetilde{\beta}^{t} \sigma L_{0}-\varkappa \widetilde{\beta}^{t} \sigma+\tilde{\varkappa} \beta^{t} \sigma-\beta^{t} \sigma \omega_{0}-\nu \alpha^{t} \sigma \omega_{1}\right) \alpha \\
& =\left(\widetilde{\beta}^{t} \sigma L_{0} \alpha-\varkappa \widetilde{\beta}^{t} \sigma \alpha\right)+\left(\tilde{\varkappa} \beta^{t} \sigma \alpha-\beta^{t} \sigma \omega_{0} \alpha\right)-\nu \alpha^{t} \sigma \omega_{1} \alpha .
\end{aligned}
$$

Ввиду (А.7), (А.3) и (А.4) это обращается в нуль.

Член нулевого порядка: надо доказать соотношение (А.7) для $L^{\prime}$. Мы имеем

$$
L_{0}^{\prime}=\left[\omega_{-1}, L_{1}\right]+\left[\omega_{0}, L_{0}\right]+\left[\omega_{1}, L_{-1}\right]+\left[\omega_{2}, L_{-2}\right]+L_{1} .
$$

Для первой скобки имеем

$$
\begin{aligned}
{\left[\omega_{-1}, L_{1}\right] \alpha } & =\left(\alpha \widetilde{\beta}^{t}+\widetilde{\beta} \alpha^{t}\right) \sigma L_{1} \alpha-L_{1}\left(\alpha \widetilde{\beta}^{t}+\widetilde{\beta} \alpha^{t}\right) \sigma \alpha \\
& =\alpha\left(\widetilde{\beta}^{t} \sigma L_{1} \alpha\right)+\widetilde{\beta}\left(\alpha^{t} \sigma L_{1} \alpha\right)-L_{1} \alpha\left(\widetilde{\beta}^{t} \sigma \alpha\right)+\widetilde{\beta}\left(\alpha^{t} \sigma \alpha\right) .
\end{aligned}
$$

Второй и четвертый члены обращаются в нуль ввиду (А.8) и кососимметричности $\sigma$ соответственно. В третьем члене заменяем $\widetilde{\beta}^{t} \sigma \alpha$ на 1 . Таким образом, мы получаем

$$
\left[\omega_{-1}, L_{1}\right] \alpha=\alpha\left(\widetilde{\beta}^{t} \sigma L_{1} \alpha\right)-L_{1} \alpha
$$

Очевидно,

$$
\left[\omega_{0}, L_{0}\right] \alpha=0 .
$$

Для третьей скобки имеем

$$
\begin{aligned}
{\left[\omega_{1}, L_{-1}\right] \alpha } & =\left[\omega_{1},\left(\alpha \beta^{t}+\beta \alpha^{t}\right) \sigma\right] \alpha \\
& =\omega_{1} \alpha\left(\beta^{t} \sigma \alpha\right)+\omega_{1} \beta\left(\alpha^{t} \sigma \alpha\right)-\alpha\left(\beta^{t} \sigma \omega_{1} \alpha\right)-\beta\left(\alpha^{t} \sigma \omega_{1} \alpha\right) \\
& =-\alpha\left(\beta^{t} \sigma \omega_{1} \alpha\right) .
\end{aligned}
$$


Наконец, для четвертого члена

$$
\left[\omega_{2}, L_{-2}\right] \alpha=\nu \omega_{2} \alpha\left(\alpha^{t} \sigma \alpha\right)-\nu \alpha\left(\alpha^{t} \sigma \omega_{2} \alpha\right),
$$

где первое слагаемое, очевидно, равно нулю, и мы имеем

$$
\left[\omega_{2}, L_{-2}\right] \alpha=-\nu \alpha\left(\alpha^{t} \sigma \omega_{2} \alpha\right)
$$

Окончательно получаем

$$
L_{0}^{\prime} \alpha=\left(\widetilde{\beta}^{t} \sigma L_{1} \alpha-\beta^{t} \sigma \omega_{1} \alpha-\nu\left(\alpha^{t} \sigma \omega_{2} \alpha\right)\right) \alpha .
$$

Член порядка 1: мы должны показать, что для $L^{\prime}$ выполнено соотношение (А.8): $\alpha^{t} \sigma L_{1}^{\prime} \alpha=0$. Мы имеем

$$
L_{1}^{\prime}=2 L_{2}+\left[\omega_{-1}, L_{2}\right]+\left[\omega_{0}, L_{1}\right]+\left[\omega_{1}, L_{0}\right]+\left[\omega_{2}, L_{-1}\right]+\left[\omega_{3}, L_{-2}\right] .
$$

Для каждого коммутатора $[\cdot, \cdot]$ в этом выражении мы вычисляем соответствующее произведение $\alpha^{t} \sigma[\cdot, \cdot] \alpha$ и получаем

$$
\begin{aligned}
\alpha^{t} \sigma\left[\omega_{-1}, L_{2}\right] \alpha= & \alpha^{t} \sigma\left[\left(\alpha \widetilde{\beta}^{t}+\widetilde{\beta} \alpha^{t}\right) \sigma, L_{2}\right] \alpha \\
= & \alpha^{t} \sigma\left(\alpha \widetilde{\beta}^{t}+\widetilde{\beta} \alpha^{t}\right) \sigma L_{2} \alpha-\alpha^{t} \sigma L_{2}\left(\alpha \widetilde{\beta}^{t}+\widetilde{\beta} \alpha^{t}\right) \sigma \alpha \\
= & \left(\alpha^{t} \sigma \alpha\right) \widetilde{\beta}^{t} \sigma L_{2} \alpha+\left(\alpha^{t} \sigma \widetilde{\beta}\right) \alpha^{t} \sigma L_{2} \alpha-\alpha^{t} \sigma L_{2} \alpha\left(\widetilde{\beta}^{t} \sigma \alpha\right) \\
& \quad-\alpha^{t} \sigma L_{2} \widetilde{\beta}\left(\alpha^{t} \sigma \alpha\right) \\
= & -2 \alpha^{t} \sigma L_{2} \alpha
\end{aligned}
$$

(ввиду соотношений $\alpha^{t} \sigma \alpha=0$ и (А.2)). Результат сократится с соответствующим вкладом слагаемого $2 L_{2}$ в вышеприведенном выражении для $L_{1}^{\prime}$.

Далее,

$$
\begin{aligned}
\alpha^{t} \sigma\left[\omega_{0}, L_{1}\right] \alpha & =\left(\alpha^{t} \sigma \omega_{0}\right) L_{1} \alpha-\alpha^{t} \sigma L_{1}\left(\omega_{0} \alpha\right) \\
& =-2 \widetilde{\varkappa}\left(\alpha^{t} \sigma L_{1} \alpha\right) \\
& =0, \\
\alpha^{t} \sigma\left[\omega_{1}, L_{0}\right] \alpha & =\alpha^{t} \sigma \omega_{1}\left(L_{0} \alpha\right)-\left(\alpha^{t} \sigma L_{0}\right) \omega_{1} \alpha \\
& =2 \varkappa\left(\alpha^{t} \sigma \omega_{1} \alpha\right) \\
& =0, \\
\alpha^{t} \sigma\left[\omega_{2}, L_{-1}\right] \alpha & =\alpha^{t} \sigma \omega_{2}\left(\alpha \beta^{t}+\beta \alpha^{t}\right) \sigma \alpha-\alpha^{t} \sigma\left(\alpha \beta^{t}+\beta \alpha^{t}\right) \sigma \omega_{2} \alpha \\
& =\alpha^{t} \sigma \omega_{2} \alpha\left(\beta^{t} \sigma \alpha\right)+\alpha^{t} \sigma \omega_{2} \beta\left(\alpha^{t} \sigma \alpha\right)-\left(\alpha^{t} \sigma \alpha\right) \beta^{t} \sigma \omega_{2} \alpha-\left(\alpha^{t} \sigma \beta\right) \alpha^{t} \sigma \omega_{2} \alpha \\
& =0, \\
\alpha^{t} \sigma\left[\omega_{3}, L_{-2}\right] \alpha & =\nu \alpha^{t} \sigma \omega_{3} \alpha\left(\alpha^{t} \sigma \alpha\right)-\nu\left(\alpha^{t} \sigma \alpha\right) \alpha^{t} \sigma \omega_{3} \alpha \\
& =0 .
\end{aligned}
$$

Следовательно,

$$
\alpha^{t} \sigma L_{1}^{\prime} \alpha=0
$$




\section{Список литературы}

[1] И. М. Кричевер, О.К. Шейнман, “Алгебры операторов Лакса”, Функи. анализ и его прил., 41:4 (2007), 46-59; англ. пер.: I. M. Krichever, O. K. Sheйnman, "Lax operator algebras", Funct. Anal. Appl., 41:4 (2007), 284-294; arXiv: math/0701648.

[2] I. M. Krichever, "Vector bundles and Lax equations on algebraic curves", Comm. Math. Phys., 229:2 (2002), 229-269.

[3] И. М. Кричевер, С.П. Новиков, "Голоморфные расслоения над алгебраическими кривыми и нелинейные уравнения", УМH, 35:6 (1980), 47-68; англ. пер.: I. M. Krichever, S.P. Novikov, "Holomorphic bundles on algebraic curves and nonlinear equations", Russian Math. Surveys, 35:6 (1980), 53-79.

[4] А.Н. Тюрин, "Классификация векторных расслоений над алгебраической кривой произвольного рода", Изв. АН СССР. Сер. матем., 29:3 (1965), 657-688; англ. пер.: A. N. Tyurin, "Classification of vector bundles over an algebraic curve of arbitrary genus", Amer. Math. Soc. Transl. Ser. 2, 63, Amer. Math. Soc., Providence, RI, 1967, 245-279.

[5] О.К. Шейнман, Алгебры Кричевера-Новикова, их представления и приложения в геометрии и математической физике, Современные проблемы математики, 10, МИАН, М., 2007.

[6] O.K. Sheinman, "Affine Krichever-Novikov algebras, their representations and applications", Geometry, topology, and mathematical physics, S. P. Novikov's seminar (Moscow, 2002-2003), Amer. Math. Soc. Transl. Ser. 2, 212, Amer. Math. Soc., Providence, RI, 2004, 297-316; arXiv: math/0304020.

[7] И. М. Кричевер, С.П. Новиков, “Алгебры типа Вирасоро, римановы поверхности и структуры теории солитонов", Функи. анализ и его прил., 21:2 (1987), 46-63; англ. пер.: I. M. Krichever, S. P. Novikov, "Algebras of Virasoro type, Riemann surfaces and structures of the theory of solitons", Funct. Anal. Appl., 21:2 (1987), $126-142$.

[8] И. М. Кричевер, С. П. Новиков, “Алгебры типа Вирасоро, римановы поверхности и струны в пространстве Минковского”, Функи. анализ и его прил., 21:4 (1987), 47-61; англ. пер.: I. M. Krichever, S. P. Novikov, "Virasoro-type algebras, Riemann surfaces and strings in Minkowsky space", Funct. Anal. Appl., 21:4 (1987), 294-307.

[9] И. М. Кричевер, С.П. Новиков, “Алгебры типа Вирасоро, тензор энергии-импульса и операторные разложения на римановых поверхностях", Функи. анализ и его прил., 23:1 (1989), 24-40; англ. пер.: I. M. Krichever, S. P. Novikov, "Algebras of Virasoro type, energy-momentum tensor, and decomposition operators on Riemann surfaces", Funct. Anal. Appl., 23:1 (1989), 19-33.

[10] М. Шлихенмайер, О.К. Шейнман, "Теория Весса-Зумино-Виттена-Новикова, уравнения Книжника-Замолодчикова и алгебры Кричевера-Новикова", УМН, 54:1 (1999), 213-250; англ. пер.: M. Schlichenmaier, O. K. Sheinman, "Wess-Zumino-Witten-Novikov theory, Knizhnik-Zamolodchikov equations, and KricheverNovikov algebras I", Russian Math. Surveys, 54:1 (1999), 213-249.

[11] М. Шлихенмайер, О.К. Шейнман, "Уравнения Книжника-Замолодчикова для положительного рода и алгебры Кричевера-Новикова", УМН, 59:4 (2004), 147-180; англ. пер.: M. Schlichenmaier, O. K. Sheinman, "Knizhnik-Zamolodchikov equations for positive genus and Krichever-Novikov algebras", Russian Math. Surveys, 59:4 (2004), 737-770.

[12] В. Г. Кац, "Простые неприводимые градуированные алгебры Ли конечного роста", Изв. АН СССР. Сер. матем., 32:6 (1968), 1323-1367; англ. пер.: V. G. Кас, "Simple irreducible graded Lie algebras of finite growth", Math. USSR-Izv., 2:6 (1968), 1271-1311.

[13] R. V. Moody, "Euclidean Lie algebras", Canad. J. Math., 21 (1969), 1432-1454. 
[14] H. Garland, "The arithmetic theory of loop groups", Inst. Hautes Études Sci. Publ. Math., 1980, № 52, 5-136.

[15] В. Кац, Бесконечномерные алгебры Ли, Мир, М., 1993; пер. с англ.: V. G. Кас, Infinite-dimensional Lie algebras, 3rd ed., Cambridge Univ. Press, Cambridge, 1990.

[16] Ch. Kassel, "Kähler differentials and coverings of complex simple Lie algebras extended over a commutative algebra", J. Pure Appl. Algebra, 34:2-3 (1984), 265-275.

[17] О.К. Шейнман, "Эллиптические аффинные алгебры Ли”, Функи. анализ и его прил., 24:3 (1990), 51-61; англ. пер.: O. K. Sheinman, "Elliptic affine Lie algebras", Funct. Anal. Appl., 24:3 (1990), 210-219.

[18] О.К. Шейнман, “Аффинные алгебры Ли на римановых поверхностях", Функи. анализ и его прил., 27:4 (1993), 54-62; англ. пер.: О. K. Sheinman, "Affine Lie algebras on Riemann surfaces", Funct. Anal. Appl., 27:4 (1993), 266-272.

[19] M. Schlichenmaier, O.K. Sheinman, "The Sugawara construction and Casimir operators for Krichever-Novikov algebras", J. Math. Sci. (New York), 92:2 (1998), 3807-3834; arXiv: q-alg/9512016.

[20] M. Schlichenmaier, "Higher genus affine algebras of Krichever-Novikov type", Mosc. Math. J., 3:4 (2003), 1395-1427.

[21] M. Schlichenmaier, "Local cocycles and central extensions for multi-point algebras of Krichever-Novikov type", J. Reine Angew. Math., 559 (2003), 53-94.

[22] M. Schlichenmaier, O.K. Sheinman, "Central extensions of Lax operator algebras. The multi-point case" (to appear).

[23] I. M. Krichever, "Isomonodromy equations on algebraic curves, canonical transformations and Whitham equations", Mosc. Math. J., 2:4 (2002), 717-752; arXiv: hep-th/0112096.

[24] M. Schlichenmaier, "Krichever-Novikov algebras for more than two points", Lett. Math. Phys., 19:2 (1990), 151-165.

[25] M. Schlichenmaier, "Krichever-Novikov algebras for more than two points: explicit generators", Lett. Math. Phys., 19:4 (1990), 327-336.

[26] M. Schlichenmaier, "Central extensions and semi-infinite wedge representations of Krichever-Novikov algebras for more than two points", Lett. Math. Phys., 20:1 (1990), 33-46.

[27] M. Schlichenmaier, Verallgemeinerte Krichever-Novikov Algebren und deren Darstellungen, Ph. D. Thesis, Universität Mannheim, Mannheim, 1990.

М. Шлихенмайер (M. Schlichenmaier)

Поступила в редакцию

University of Luxembourg 16.06 .2008

E-mail: martin.schlichenmaier@uni.lu

O. К. Шейнман (О... Sheinman)

Математический институт им. В. А. Стеклова РАН

E-mail: sheinman@mi.ras.ru 